\title{
PRESENTATIONS OF CATEGORIES OF MODULES USING THE CAUTIS-KAMNITZER-MORRISON PRINCIPLE
}

\author{
GIULIAN WIGGINS
}

\begin{abstract}
We use duality theorems to obtain presentations of some categories of modules. To derive these presentations we generalize a result of Cautis-KamnitzerMorrison [CKM14]:

Let $\mathfrak{g}$ be a reductive Lie algebra, and $A$ an algebra, both over $\mathbb{C}$. Consider a $(\mathfrak{g}, A$ )-bimodule $P$ in which

(a) $P$ has a multiplicity free decomposition into irreducible $(\mathfrak{g}, A)$-bimodules.

(b) $P$ is "saturated" i.e. for any irreducible $\mathfrak{g}$-module $V$, if every weight of $V$ is a weight of $P$, then $V$ is a submodule of $P$.

We show that statements (a) and (b) are necessary and sufficient conditions for the existence of an isomorphism of categories between the full subcategory of $\mathcal{R} e p A$ whose objects are $\mathfrak{g}$-weight spaces of $P$, and a quotient of the category version of Lusztig's idempotented form, $\dot{\mathcal{U}} \mathfrak{g}$, formed by setting to zero all morphisms factoring through a collection of objects in $\dot{\mathcal{U}} \mathfrak{g}$ depending on $P$. This is essentially a categorical version of the identification of generalized Schur algebras with quotients of Lusztig's idempotented forms given in [Dot03].

Applied to Schur-Weyl Duality we obtain a diagrammatic presentation of the full subcategory of $\mathcal{R} e p S_{d}$ whose objects are direct sums of permutation modules, as well as an explicit description of the $\otimes$-product of morphisms between permutation modules. Applied to Brauer-Schur-Weyl Duality we obtain diagrammatic presentations of subcategories of $\mathcal{R} e p \mathcal{B}_{d}^{(-2 n)}$ and $\mathcal{R} e p \mathcal{B}_{r, s}^{(n)}$ whose Karoubi completion is the whole of $\mathcal{R} e p \mathcal{B}_{d}^{(-2 n)}$ and $\mathcal{R} e p \mathcal{B}_{r, s}^{(n)}$ respectively.
\end{abstract}

\section{Contents}

1. Introduction

1.1. A diagrammatic presentation of $\mathcal{P} \operatorname{erm}\left(S_{d}\right)$

1.2. The Kronecker product of permutation modules

1.3. Acknowledgements

2. Cautis-Kamnitzer-Morrison Principle

3. A Diagrammatic Presentation of Perm

3.1. The category of $\mathcal{P}$ erm-spiders

3.2. The category $\dot{\mathcal{U}} \mathfrak{g l}$

3.3. Schur-Weyl Duality and the CKM Principle 13

3.4. Proof of Theorem 3.1.1 15

4. A Tabloid-Theoretic Description of the Generating Morphisms of $\dot{\mathcal{S}}(n, d) \quad 18$

5. Symmetric Monoidal Structure on $\mathcal{P}$ erm 20

5.1. A diagrammatic presentation of $\mathcal{P} r m$ 
6. The Monoidal Structure on $\operatorname{Prm}\left(S_{d}\right) \quad 24$

6.1. Examples 26

6.2. Proof of Theorem 6.0.1 28

7. Brauer Algebras and the CKM Principle 29

7.1. Schur-Weyl Duality in types C and D 29

7.2. A pre-Karoubi presentation of $\mathcal{R e p}_{d}^{(-2 n)} \quad 31$

7.3. Mixed Schur-Weyl Duality and the CKM principle 35

References 36

\section{INTRODUCTION}

Let $\mathfrak{g}$ denote a reductive Lie algebra over $\mathbb{C}$. Let $\mathfrak{g}=\mathfrak{s} \oplus \mathfrak{a}$ be the decomposition of $\mathfrak{g}$ into semisimple and abelian parts. Let $\Delta=\left\{\alpha_{1}, \ldots, \alpha_{n}\right\}$ be a choice of positive simple roots for $\mathfrak{s}$, and $\Lambda$ the integral weight lattice for $\mathfrak{g}$. Let $\left\{e_{i}, f_{i}, h_{i}\right\}_{i=1, \ldots, n}$ be the Chevalley generators of $\mathfrak{s}$ with respect to $\Delta$, and $\left(a_{i j}\right)$ the Cartan matrix of $\mathfrak{s}$ i.e. $h_{i}=\alpha_{i}^{\vee}$ and $a_{i j}=\alpha_{i}\left(h_{j}\right)$.

Consider the idempotented form of the enveloping algebra $U \mathfrak{g}$, which we regard as the $\mathbb{C}$-linear category, $\dot{\mathcal{U}} \mathfrak{g}$, with

- Objects: Integral weights $\lambda \in \Lambda$.

- Morphisms: Let $1_{\lambda}$ denote the identity on $\lambda$. Let $1_{\mu} \dot{\mathcal{U}} \mathfrak{g} 1_{\lambda}$ denote the space of morphisms from $\lambda$ to $\mu$. The morphisms are generated by $E_{i} 1_{\lambda} \in 1_{\lambda+\alpha_{i}} \dot{\mathcal{U}} \mathfrak{g} 1_{\lambda}$ and $F_{i} 1_{\lambda} \in 1_{\lambda-\alpha_{i}} \dot{\mathcal{U}} \mathfrak{g} 1_{\lambda}$ for $1 \leq i \leq n$. These morphisms satisfy the relations

$$
\begin{array}{rlrl}
E_{i} F_{i} 1_{\lambda} & =F_{i} E_{i} 1_{\lambda}+\lambda\left(h_{i}\right) 1_{\lambda} & \\
E_{i} F_{j} 1_{\lambda} & =F_{j} E_{i} 1_{\lambda} & & \text { if } i \neq j \\
\operatorname{ad}\left(E_{j}\right)^{1-a_{i j}} E_{i} 1_{\lambda} & =0 & & \text { and likewise with } F \text { replacing } E
\end{array}
$$

This paper only considers $\mathfrak{g}$-modules with an integral weight space decomposition. For this reason we henceforth use the term $\mathfrak{g}$-module to only refer to those $\mathfrak{g}$-modules with an integral weight space decomposition.

Let $V e c_{\mathbb{C}}$ denote the category of vector spaces over $\mathbb{C}$. Any $\mathfrak{g}$-module $P$ is equivalent to a $\mathbb{C}$-linear functor $\mathcal{F}_{P}: \dot{\mathcal{U}} \mathfrak{g} \rightarrow V e c_{\mathbb{C}}$ mapping objects $\lambda \in \Lambda$ to the $\lambda$-weight space of $P, P_{\lambda}$, and each morphism $E_{i} 1_{\lambda} \in 1_{\lambda+\alpha_{i}} \dot{\mathcal{U}} \mathfrak{g} 1_{\lambda}$ to the map

$$
P_{\lambda} \rightarrow P_{\lambda+\alpha_{i}}: v \mapsto e_{i} v
$$

and likewise with the $F_{i} 1_{\lambda}$.

For any $\mathfrak{g}$-module $P$ write $\Pi(P)$ for the set of weights of $P$. Define $\dot{\mathcal{U}}^{P} \mathfrak{g}$ to be the $\mathbb{C}$-linear category defined

- Objects: Weights of $P$.

- Morphisms: The space, $1_{\mu} \dot{\mathcal{U}}^{P} \mathfrak{g} 1_{\lambda}$, of morphisms from $\lambda$ to $\mu$ is defined as the quotient of $1_{\mu} \dot{\mathcal{U}} \mathfrak{g} 1_{\lambda}$ by the space of morphisms $f \in 1_{\mu} \dot{\mathcal{U}} \mathfrak{g} 1_{\lambda}$ that factor through an object $\nu \notin \Pi(P)$. 
Doty-Giaquinto-Sullivan [DGS09] introduced the term saturated $\mathfrak{g}$-module to describe those $\mathfrak{g}$-modules $P$ with the property that for every $\mathfrak{g}$-dominant weight $\lambda \in$ $\Pi(P)$, the irreducible module of highest weight $\lambda$ is a submodule of $P$.

This paper gives two main results regarding saturated $\mathfrak{g}$-modules:

- (Doty Criterion, Lemma 2.0.3) The functor $\dot{\mathcal{U}}^{P} \mathfrak{g} \rightarrow V e c_{\mathbb{C}}$ induced by $\mathcal{F}_{P}$ : $\dot{\mathcal{U}} \mathfrak{g} \rightarrow V e c_{\mathbb{C}}$ is faithful if and only if $P$ is saturated.

- (CKM Principle, Theorem 2.0.4) Let $A$ be an algebra over $\mathbb{C}$, and $P$ a finite dimensional $(\mathfrak{g}, A)$-bimodule with multiplicity free decomposition

$$
P=\bigoplus_{i \in \chi} V^{i} \otimes M^{i}
$$

where the $V^{i}$ are irreducible $\mathfrak{g}$-modules, and the $M^{i}$ are irreducible $A$-modules. If $P$ is a saturated $\mathfrak{g}$-module, then the category $\dot{\mathcal{U}}^{P} \mathfrak{g}$ is isomorphic to the full subcategory of $\mathcal{R} e p A$ whose objects are the $\mathfrak{g}$-weight spaces of $P$. Moreover, if $A$ is semisimple and acts on $P$ faithfully then $\mathcal{R e p} A$ is equivalent to the Karoubi completion of the additive closure of $\dot{\mathcal{U}}^{P} \mathfrak{g}$ (Corollary 2.0.5).

Remark. The Doty Criterion should be viewed as a categorical version of the correspondence between generalized Schur algebras and certain quotients of Lusztig's idempotented forms given in ([Dot03], Theorem 4.2). Indeed the Doty Criterion gives a condition for an isomorphism of algebras

$$
\bigoplus_{\lambda, \mu \in \Pi(P)} 1_{\mu} \dot{\mathcal{U}}^{P} \mathfrak{g} 1_{\lambda} \cong \bigoplus_{\lambda, \mu \in \Pi(P)} \mathcal{F}_{P}\left(1_{\mu} \dot{\mathcal{U}}^{P} \mathfrak{g} 1_{\lambda}\right) .
$$

Whenever $P$ is saturated, the right hand side of (1.5) is the generalized Schur algebra $\mathbf{S}(\Pi(P))$ as defined in [Don86]. Moreover this isomorphism is exactly that given by ([Dot03], Theorem 4.2).

The CKM Principle is the special case of the Doty Criterion in the case where $P$ is a $(\mathfrak{g}, A)$-bimodule. Cautis-Kamnitzer-Morrison [CKM14] proved this result for (the quantum analog of ) the $U\left(\mathfrak{g l}_{m}\right) \otimes U\left(\mathfrak{s l}_{n}\right)$-module $\bigwedge^{\bullet}\left(\mathbb{C}^{n} \otimes \mathbb{C}^{m}\right)$. This has a multiplicity free decomposition by skew-Howe duality. From this they give a presentation of the full subcategory of $\mathcal{R} e p \mathfrak{s l}_{n} \otimes$-generated by the fundamental representations of $\mathfrak{s l}_{n}$.

In Section 2 we give elementary proofs of the Doty Criterion and CKM Principle.

The following multiplicity-free commuting actions are saturated ([DGS09], Section $6)$,

$$
\begin{aligned}
& \mathfrak{g l}_{n} \curvearrowright \bigotimes^{d} \mathbb{C}^{n} \curvearrowleft \mathbb{C}\left[S_{d}\right] \\
& \mathfrak{s p}_{2 n} \curvearrowright \bigotimes^{d} \mathbb{C}^{2 n} \curvearrowleft \mathcal{B}_{d}^{(-2 n)} \\
& \mathfrak{o}_{2 n} \curvearrowright \bigotimes^{d} \mathbb{C}^{2 n} \curvearrowleft \mathcal{B}_{d}^{(2 n)} \\
& \mathfrak{g l}_{n} \curvearrowright \bigotimes^{r} \mathbb{C}^{n} \otimes \bigotimes^{s} \mathbb{C}^{* n} \curvearrowleft \mathcal{B}_{r, s}^{(n)}
\end{aligned}
$$

where $\mathcal{B}_{d}^{\delta}$ is the Brauer algebra over $\mathbb{C}$ spanned by $d$-diagrams parametrized by $\delta$ (as defined in [Bra37]), and $\mathcal{B}_{r, s}^{(n)} \subset \mathcal{B}_{r+s}^{(n)}$ is the walled Brauer algebra (as defined in 
[BCHLLS94]). These definitions and the commuting actions in (1.7), (1.8), (1.9) are recalled in Section 7.

Say that a category, $\mathcal{D}$, is a pre-Karoubi subcategory of a category, $\mathcal{C}$, if $\mathcal{C}$ is equivalent to the additive closure of the Karoubi completion of $\mathcal{D}$. In this case we call any presentation of $\mathcal{D}$ a pre-Karoubi presentation of $\mathcal{C}$.

If $n \geq d$ then the right action in (1.6) is faithful. We use the CKM principle to give a pre-Karoubi presentation of $\mathcal{R} e p S_{d}$ (Theorem 3.1.1).

If $2 n \geq d-1$ then the right actions in (1.7) and (1.8) are faithful [Bro56]. We give a pre-Karoubi presentation of $\mathcal{R} e p \mathcal{B}_{d}^{(-2 n)}$ for $2 n \geq d-1$ (Proposition 7.2.1).

If $n \geq r+s$ then the right action in (1.9) is faithful [BCHLLS94]. We give a pre-Karoubi presentation of $\mathcal{R} \operatorname{ep} \mathcal{B}_{r, s}^{(n)}$ for $n \geq r+s$ (Proposition 7.3.1).

1.1. A diagrammatic presentation of $\operatorname{Perm}\left(S_{d}\right)$. Let us explain in more detail the pre-Karoubi presentation of $\mathcal{R} e p S_{d}$. Given a sequence $\lambda=\left(\lambda_{1}, \ldots, \lambda_{n}\right) \in \mathbb{Z}^{n}$ and $d \in \mathbb{Z}$, write $\lambda \vDash d$ if $\lambda$ is a composition of $d$. For $\lambda \vDash d$ denote the Young subgroup

$$
S_{\lambda}:=S_{\left\{1, \ldots, \lambda_{1}\right\}} \times S_{\left\{\lambda_{1}+1, \ldots, \lambda_{1}+\lambda_{2}\right\}} \times \cdots \times S_{\left\{\lambda_{1}+\cdots+\lambda_{n-1}+1, \ldots, d\right\}}
$$

We give a diagrammatic presentation of the full subcategory, $\operatorname{Perm}\left(S_{d}\right)$, of $\mathcal{R}$ ep $S_{d}$ whose objects are the right $\mathbb{C}\left[S_{d}\right]$ modules $M^{\lambda}=\mathbb{C}\left[S_{\lambda} \backslash S_{d}\right]$, where $\lambda \vDash d$ (Theorem 3.1.1). Indeed every $M^{\lambda}, \lambda \vDash d$, is isomorphic in $\mathcal{R e p} S_{d}$ to a $\mathfrak{g l}_{n}$-weight space of $\bigotimes^{d} \mathbb{C}^{d}$. By applying the CKM-principle and the diagrammatic calculus developed in [CKM14], we derive a presentation of $\mathcal{P} \operatorname{erm}\left(S_{d}\right)$. We outline these results here.

First define the category $\mathcal{P}$ erm with

- Objects: The permutation modules $M_{d}^{\lambda}:=\mathbb{C}\left[S_{\lambda} \backslash S_{d}\right]$ for all $d \in \mathbb{N}$ and $\lambda \vDash d$.

- Morphisms:

$$
\operatorname{Hom}\left(M_{d}^{\lambda}, M_{d^{\prime}}^{\mu}\right)= \begin{cases}\operatorname{Hom}_{S_{d}}\left(M_{d}^{\lambda}, M_{d}^{\mu}\right) & \text { if } d=d^{\prime} \\ 0 & \text { otherwise }\end{cases}
$$

Remark. Perm is the full subcategory of $\bigoplus_{d=0}^{\infty} \mathcal{R} e p S_{d}$ whose objects are the $M_{d}^{\lambda}$. We omit the subscript in $M_{d}^{\lambda}$ when the value of $d$ is clear from the context.

It is more natural from the diagrams perspective to first give a diagrammatic presentation of $\mathcal{P}$ erm, and then consider $\mathcal{P} \operatorname{Prm}\left(S_{d}\right)$ as a full subcategory of $\mathcal{P}$ erm. For this we define a monoidal product on $\mathcal{P} e r m$.

The category $\bigoplus_{d=0}^{\infty} \mathcal{R} e p S_{d}$ has a monoidal product defined on direct summands by the bifunctor

$$
\cdot \circ \cdot=\operatorname{Ind}_{S_{d} \times S_{d^{\prime}}}^{S_{d+d^{\prime}}}(\cdot \otimes \cdot): \mathcal{R} e p S_{d} \times \mathcal{R} e p S_{d^{\prime}} \rightarrow \mathcal{R} e p S_{d+d^{\prime}}
$$

This restricts to a monoidal product on $\mathcal{P}$ erm since

$$
M_{d}^{\lambda} \circ M_{d^{\prime}}^{\mu}=\left(\mathbb{C}\left[S_{\lambda} \backslash S_{d}\right] \otimes \mathbb{C}\left[S_{\mu} \backslash S_{d^{\prime}}\right]\right) \otimes_{\mathbb{C}\left[S_{d} \times S_{d^{\prime}}\right]} \mathbb{C}\left[S_{d+d^{\prime}}\right]=\mathbb{C}\left[S_{\lambda} \times S_{\mu} \backslash S_{d+d^{\prime}}\right]=M_{d+d^{\prime}}^{(\lambda, \mu)}
$$

where $(\lambda, \mu)$ is the concatenation of the sequences $\lambda$ and $\mu$. We regard Perm as a monoidal category with the monoidal product $\cdot \circ \cdot$. It will be shown (Theorem 3.1.1) 
that the morphisms of Perm are generated (as a monoidal $\mathbb{C}$-linear category) by the morphisms:

For $k, l$ nonnegative integers, and $G=S_{k, l} \backslash S_{k+l}$ :

$$
\begin{array}{ll}
\nabla_{k, l}: M^{k, l} \rightarrow \mathbb{C}=M^{k+l} ; \sum_{g \in G} k_{g} g \rightarrow \sum_{g \in G} k_{g} & \text { for } k_{g} \in \mathbb{C} \\
\Delta_{k, l}: \mathbb{C}=M^{k+l} \rightarrow M^{k, l} ; 1 \rightarrow \sum_{g \in G} g=\frac{1}{k ! l !} \sum_{g \in S_{k+l}} S_{k, l} g &
\end{array}
$$

We depict these morphisms diagrammatically

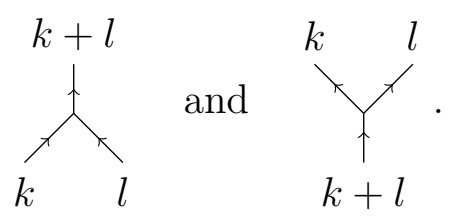

with composition drawn by vertical juxtaposition, and the o-product drawn by horizontal juxtaposition. Theorem 3.1.1 states that $\mathcal{P}$ erm is the monoidal $\mathbb{C}$-linear category o-generated by the $\nabla_{k, l}, \Delta_{k, l}$ modulo the relations (3.1), (3.2), (3.3), (3.4), and any planar isotopy that keeps the edges oriented upwards.

A presentation of $\mathcal{P} \operatorname{erm}\left(S_{d}\right)$ can be obtained from this presentation by restricting to diagrams in which the numbers along the bottom (and top) sum to $d$. In particular, $\operatorname{Perm}\left(S_{d}\right)$ is generated by the morphisms, for $\lambda=\left(\lambda_{1}, \ldots, \lambda_{n}\right) \vDash d$,

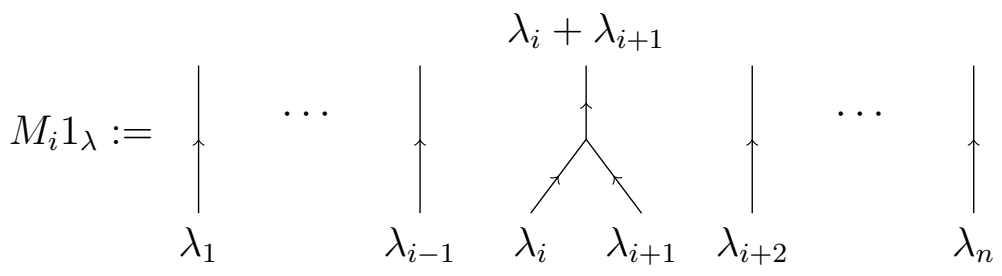

and

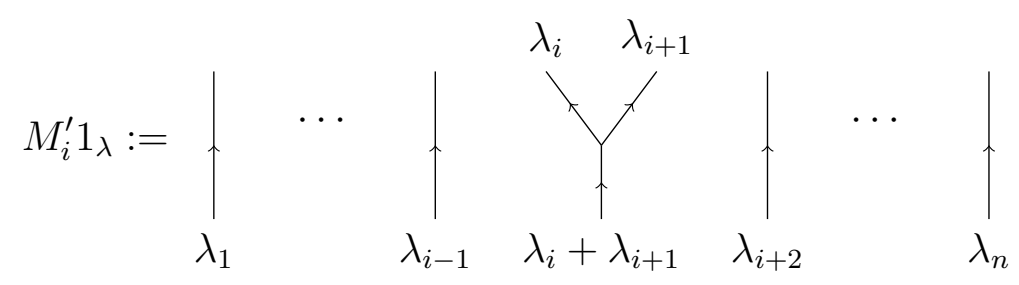

These are defined explicitly, for $\lambda^{\prime}=\left(\lambda_{1}, \ldots, \lambda_{i}+\lambda_{i+1}, \ldots, \lambda_{n}\right)$,

$$
\begin{aligned}
& M_{i} 1_{\lambda}: M^{\lambda} \rightarrow M^{\lambda^{\prime}} ; S_{\lambda} g \mapsto S_{\lambda^{\prime}} g \\
& M_{i}^{\prime} 1_{\lambda}: M^{\lambda^{\prime}} \rightarrow M^{\lambda} ; S_{\lambda^{\prime}} g \mapsto \frac{1}{\lambda_{i} ! \lambda_{i+1} !} \sum_{h \in S_{\left\{\lambda_{i-1}+1, \ldots, \lambda_{i+1}\right\}}} S_{\lambda} h g
\end{aligned}
$$


The category $\mathcal{P}$ erm is symmetric monoidal, with the braiding isomorphism $M^{k, l} \rightarrow$ $M^{l, k}$ defined diagrammatically (Theorem 5.1.1):

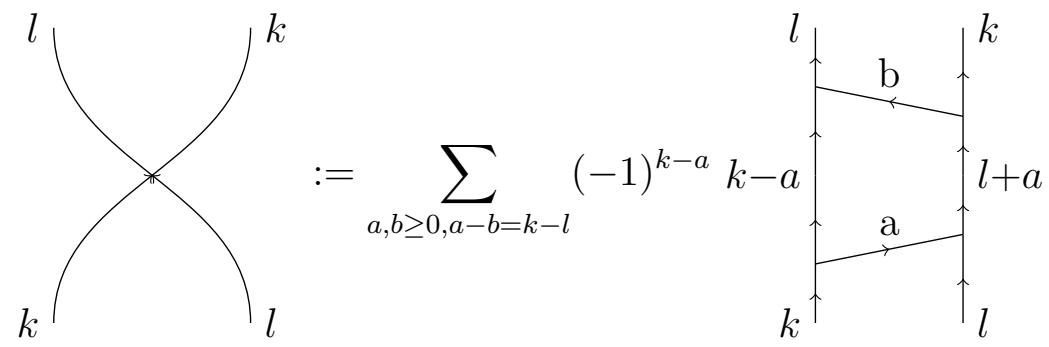

1.2. The Kronecker product of permutation modules. It is is known that the tensor product of two permutation modules of $S_{d}$ decomposes as a direct sum of permutation modules of $S_{d}$ ([JK81], lemma 2.9.16). In Section 6 we recall this decomposition, and give an explicit description of the $\otimes$-product of morphisms between permutation modules, in terms of our generating morphisms.

1.3. Acknowledgements. Part of this research was undertaken for an honours thesis at the University of Sydney, under the supervision of Dr. Oded Yacobi.

I thank my mentor Dr. Oded Yacobi for suggesting this problem to me, and for the continual guidance and feedback in the research and writing of this paper.

I have also benefitted from Cautis, Kamnitzer, Morrison uploading the source file for [CKM14] onto the arXiv. Indeed many of the diagrams here use their macros.

\section{Cautis-Kamnitzer-Morrison Principle}

In this section we prove the CKM principle. Recall we use the term $\mathfrak{g}$-module to just refer to $\mathfrak{g}$-modules with an integral weight space decomposition. Let $\Lambda$ denote the integral weights of $\mathfrak{g}$. For any $\mathfrak{g}$-module $V$, write $\Pi(V)$ for the set of weights of $V$, and $\Pi^{+}(V)$ for the set of $\mathfrak{g}$-dominant weights of $V$. For any $\mathfrak{g}$-dominant weight $\lambda \in \Lambda$, write $V^{\lambda}$ for the irreducible $\mathfrak{g}$-module of highest weight $\lambda$.

Say that a $\mathfrak{g}$-module $P$ is saturated (for $\mathfrak{g}$ ) if $\left\{V^{\lambda}\right\}_{\lambda \in \Pi^{+}(P)}$ is the set of irreducible $\mathfrak{g}$-submodules of $P$ up to isomorphism.

Lemma 2.0.1. A finite dimensional $\mathfrak{g}$-module $P$ is saturated if and only if for any irreducible $\mathfrak{g}$-module $V, \Pi(V) \subseteq \Pi(P)$ implies $V$ is a submodule of $P$.

Proof. Define the usual partial order on $\Lambda: \lambda \geq \mu$ if $\lambda-\mu$ is a sum of positive roots. Let $\mathcal{W}$ be the Weyl group of $\mathfrak{g}$. If $\lambda \in \Lambda$ is $\mathfrak{g}$-dominant then

$$
\Pi\left(V^{\lambda}\right)=\{\mu \in \Lambda \mid \text { if } \nu \in \mathcal{W} \mu \text { then } \nu \leq \lambda\}
$$

Hence $\lambda \in \Pi^{+}(P)$ if and only if $\Pi\left(V^{\lambda}\right) \subseteq \Pi(P)$. The result follows.

Define the algebra version of Lusztig's idempotented form,

$$
\dot{U} \mathfrak{g}:=\bigoplus_{\lambda, \mu \in \Lambda} 1_{\mu} \dot{\mathcal{U}} \mathfrak{g} 1_{\lambda}
$$


with multiplication defined by composition in $\dot{\mathcal{U}} \mathfrak{g}$. Define the two-sided ideal, $I_{P}$, of $\dot{U} \mathfrak{g}$

$$
I_{P}:=\left\langle 1_{\lambda} \mid \lambda \notin \Pi(P)\right\rangle
$$

Recall that a $\mathfrak{g}$-module $P$ is equivalent to a functor $\mathcal{F}_{P}: \dot{\mathcal{U}} \mathfrak{g} \rightarrow V e c_{\mathbb{C}}$, and this functor factors through the category $\dot{\mathcal{U}}^{P} \mathfrak{g}$ defined by setting to zero all morphisms that factor through an object $\lambda \notin \Pi(P)$. Such a functor $\mathcal{F}_{P}: \dot{\mathcal{U}} \mathfrak{g} \rightarrow V e c_{\mathbb{C}}$ is equivalent to a representation map $\dot{F}_{P}: \dot{U} \mathfrak{g} \rightarrow$ End $P$. Clearly $\dot{F}_{P}$ factors through the algebra

$$
\begin{aligned}
\dot{U}^{P} \mathfrak{g} & :=\bigoplus_{\lambda, \mu \in \Pi(P)} 1_{\mu} \dot{\mathcal{U}}^{P} \mathfrak{g} 1_{\lambda} \\
& =\dot{U} \mathfrak{g} / I_{P}
\end{aligned}
$$

It is shown in ([Dot03], Theorem 4.2) that the algebra $\dot{U}^{P} \mathfrak{g}$ is isomorphic to the generalized Schur algebra $\mathbf{S}(\Pi(P)):=\dot{U} \mathfrak{g} / \operatorname{Ker} \dot{F}_{P}$. The following lemma is shown in [Dot03] and we include our own proof for completeness.

Lemma 2.0.2. If $P$ is a finite dimensional saturated $\mathfrak{g}$-module then

(1) $\left\{V^{\lambda}\right\}_{\lambda \in \Pi^{+}(P)}$ is the set of irreducible $\dot{U}^{P} \mathfrak{g}$-modules up to isomorphism.

(2) $\dot{U}^{P} \mathfrak{g}$ is finite dimensional and semisimple.

Proof. The (irreducible) $\dot{U}^{P} \mathfrak{g}$ modules are the (irreducible) $\mathfrak{g}$-modules $V$ such that $\Pi(V) \subseteq \Pi(P)$. By Lemma 2.0.1 the irreducible $\dot{U}^{P} \mathfrak{g}$ modules are precisely the irreducible submodules $\left\{V^{\lambda}\right\}_{\lambda \in \Pi^{+}(P)}$. By the PBW theorem, and since $P$ has finitely many weights, $\dot{U}^{P} \mathfrak{g}$ is finite dimensional. Since $\mathfrak{g}$-modules are completely reducible, $\dot{U}^{P} \mathfrak{g}$ is semisimple.

The kernel of the algebra representation map $\dot{F}_{P}: \dot{U} \mathfrak{g} \rightarrow$ End $P$ is the two-sided ideal in $\dot{U} \mathfrak{g}$,

$$
\operatorname{Ker} \dot{F}_{P}:=\left\langle f \in 1_{\mu} \dot{\mathcal{U}} \mathfrak{g} 1_{\lambda} \mid \mathcal{F}_{P}(f)=0 \in \operatorname{Hom}_{\mathbb{C}}\left(P_{\lambda}, P_{\mu}\right), \lambda, \mu \in \Lambda\right\rangle
$$

The following lemma is a direct consequence of ([Dot03], Theorem 4.2). We include a more elementary proof.

Lemma 2.0.3 (Doty Criterion). A finite dimensional $\mathfrak{g}$-module $P$ is saturated if and only if $\operatorname{Ker} \dot{F}_{P}=I_{P}$.

Proof. Suppose $P$ is saturated. Clearly $I_{P} \subseteq \operatorname{Ker} \dot{F}_{P}$. To prove the converse it suffices to show that $\dot{U} \mathfrak{g} / I_{P}$ acts on $P$ faithfully. By Lemma 2.0.2,

$$
\begin{aligned}
\left\{f \in \dot{U}^{P} \mathfrak{g} \mid f P=0\right\} & =\operatorname{Rad}\left(\dot{U}^{P} \mathfrak{g}\right) & & \text { by Lemma 2.0.2 } \\
& =0 & & \text { by Lemma 2.0.2 }
\end{aligned}
$$

as required.

If $\mathrm{P}$ is not saturated then Wedderburn's theorem gives a decomposition

$$
\dot{U}^{P} \mathfrak{g}=\left(\bigoplus_{\lambda \in \Pi^{+}(P)} \operatorname{End} V^{\lambda}\right) \oplus\left(\bigoplus_{i \in \chi} \operatorname{End} W^{i}\right)
$$


where the $W^{i}$ are nonzero irreducible $\mathfrak{g}$-modules not contained in $P$ (but whose weights are weights of $P$ ). Then each End $W^{i} \subset \operatorname{Ker} \dot{F}_{P}$ but End $W^{i} \not \subset I_{P}$.

Remark. As an example of Lemma 2.0.3 take $\mathfrak{g}=\mathfrak{s l}_{2}(\mathbb{C})$ and $P=V(2)$, the irreducible $\mathfrak{s l}_{2}$-module of highest weight 2. Then $z:=E F 1_{0}+F E 1_{0}-41_{0} \in 1_{0} \dot{\mathcal{U}}_{\mathfrak{s l}} 1_{0}$ is in Ker $\dot{F}_{P}$ and not in $I_{P}$. If we instead take $P$ to be the saturated module $V(2) \oplus V(0)$ then $z \notin \operatorname{Ker} \dot{F}_{P}$ as expected.

Theorem 2.0.4 (CKM Principle). Let $A$ be an algebra over $\mathbb{C}$, and $P$ a finite dimensional $(\mathfrak{g}, A)$-bimodule that has a multiplicity free decomposition

$$
P=\bigoplus_{\lambda \in \Pi^{+}(P)} V^{\lambda} \otimes L^{\lambda}
$$

where $\left\{L^{\lambda}\right\}_{\lambda \in \Pi^{+}(P)}$ is some family of irreducible $A$-modules. Then $\dot{U}^{P} \mathfrak{g} \cong \operatorname{End}_{A} P$ and $\dot{\mathcal{U}}^{P} \mathfrak{g}$ is isomorphic to the full subcategory, $\mathcal{R} e p^{P} A$, of $\mathcal{R} e p A$ whose objects are the weight spaces of $P$.

Proof. By Lemma 2.0.3, the $\mathbb{C}$-linear functor $\mathcal{F}_{P}: \dot{\mathcal{U}} \mathfrak{g} \rightarrow V e c_{\mathbb{C}}$ factors through a faithful functor $\dot{\mathcal{U}}^{P} \mathfrak{g} \rightarrow \mathcal{R} \operatorname{ep} A$. This functor is fully-faithful since we have the following isomorphism of algebras,

$$
\begin{aligned}
\bigoplus_{\lambda, \mu \in \Pi(P)} \operatorname{Hom}_{A}\left(P_{\lambda}, P_{\mu}\right) & \cong \operatorname{End}_{A} P \\
& =\operatorname{End}_{A}\left(\bigoplus_{\lambda \in \Pi^{+}(P)} V^{\lambda} \otimes L^{\lambda}\right) \\
& \cong \bigoplus_{\lambda \in \Pi^{+}(P)} \operatorname{End}_{A}\left(V^{\lambda} \otimes L^{\lambda}\right) \\
& \cong \bigoplus_{\lambda \in \Pi^{+}(P)} \operatorname{End}^{\lambda} \otimes \operatorname{End}_{A} L^{\lambda} \\
& \cong \bigoplus_{\lambda \in \Pi^{+}(P)} \operatorname{End} V^{\lambda} \\
& \cong \dot{U}^{P} \mathfrak{g}
\end{aligned}
$$

by Schur's Lemma and multiplicity freeness

by Schur's Lemma

by Lemma 2.0.2 and Wedderburn's Theorem

The result follows.

Given a linear category $\mathcal{C}$, define the free additive category generated by $\mathcal{C}$, add- $\mathcal{C}$, to be the category whose objects are biproducts of objects in $\mathcal{C}$, as well as a zero object. Morphisms $f: A_{1} \oplus \cdots \oplus A_{n} \rightarrow B_{1} \oplus \cdots \oplus B_{m}$ in add- $\mathcal{C}$ are written as $m \times n$-matrices

$$
f=\left(\begin{array}{ccccc}
f_{11} & f_{12} & f_{13} & \ldots & f_{1 n} \\
f_{21} & f_{22} & f_{23} & \ldots & f_{2 n} \\
\vdots & \vdots & \vdots & \ddots & \vdots \\
f_{m 1} & f_{m 2} & f_{m 3} & \ldots & f_{m n}
\end{array}\right)
$$


where $f_{i j}: A_{j} \rightarrow B_{i}$ is a morphism in $\mathcal{C}$. Addition and composition of morphisms in add- $\mathcal{C}$ is given by usual matrix addition and multiplication.

The Karoubi envelope of $\mathcal{C}$ has as objects pairs $(A, e)$ where $A$ is an object in $\mathcal{C}$ and $e: A \rightarrow A$ is an idempotent morphism in $\mathcal{C}$. Morphisms $(A, e) \rightarrow(B, f)$ are morphisms $\varphi: A \rightarrow B$ in $\mathcal{C}$ such that $\varphi=f \varphi e$.

Corollary 2.0.5. Assume the same set up as Theorem 2.0.4. Suppose A is finite dimensional and semisimple. If $A$ acts on $P$ faithfully then the Karoubi envelope of add $-\dot{\mathcal{U}}^{P} \mathfrak{g}$ is equivalent to $\mathcal{R} \operatorname{ep} A$.

Proof. By the CKM Principle add- $\dot{\mathcal{U}}^{P} \mathfrak{g}$ is isomorphic to add- $\mathcal{R} e p^{P} A$, which is equivalent to the full subcategory of $\mathcal{R} e p A$ whose objects are direct sums of $\mathfrak{g}$-weight spaces of $P$. Now, $P$ has the following decompositions into $A$-modules,

$$
P=\bigoplus_{\lambda \in \Pi(P)} P_{\lambda}=\bigoplus_{\lambda \in \Pi^{+}(P)}\left(\operatorname{dim} V^{\lambda}\right) L^{\lambda} .
$$

Hence each of the $L^{\lambda}$ appear as a direct summand of one of the $P_{\lambda}$. Hence it is enough to show that $\left\{L^{\lambda}\right\}_{\lambda \in \Pi^{+}(P)}$ is the set of all irreducible $A$-modules up to isomorphism. Note that we can identify,

$$
\begin{array}{rlr}
A & \subseteq \operatorname{End}_{\mathfrak{g}}(P) & \text { since } A \text { acts faithfully on } P \\
& =\bigoplus_{\lambda \in \Pi^{+}(P)} \text { End } L^{\lambda} & \text { as in the proof of Theorem 2.0.4 } \\
& \subseteq A & \text { by Wedderburn's Theorem }
\end{array}
$$

It follows that $\left\{L^{\lambda}\right\}_{\lambda \in \Pi^{+}(P)}$ is the set of all irreducible $A$-modules up to isomorphism. The result follows.

\section{A Diagrammatic Presentation of $\mathcal{P} e r m$}

As a first application of the CKM Principle, we use Schur-Weyl duality to derive a diagrammatic presentation of the full subcategory, $\mathcal{P} e r m$, of $\bigoplus_{d=0}^{\infty} \mathcal{R} e p S_{d}$ whose objects are the permutation modules, $M^{\lambda}$, for $\lambda \vDash d, d \in \mathbb{N}$ (Theorem 3.1.1).

3.1. The category of $\mathcal{P}$ erm-spiders. Define a $\mathcal{P}$ erm-spider to be a planar diagram made up of $\mathbb{Z}_{>0}$-labelled strands whose endpoints either intersect the bottom or top of the diagram, or intersect a vertex of the form

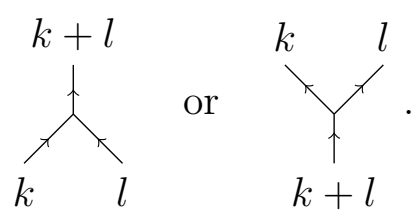

We identify $\mathcal{P}$ erm-spiders up to any planar isotopy that preserves the upwards orientation of the edges. Given two sequences $\lambda, \mu$ of integers we say that a Permspider connects $\lambda$ to $\mu$ if the sequence of edges along the bottom (respectively top) of the diagram is $\lambda$ (respectively $\mu$ ). 
We draw $\mathcal{P}$ erm-spiders with strands labelled by any integer. Strands with a 0 label are interpreted by removing the strand from the diagram. Strands with a non-positive label are zero morphisms.

Define the $\mathbb{C}$-linear monoidal category $\mathcal{S} p(\mathcal{P}$ erm $)$ :

- Objects: Finite sequences of nonnegative integers. The monoidal product on objects is given by concatenation.

- Morphisms: Morphisms from $\lambda$ to $\mu$ are $\mathbb{C}$-linear combinations of Permspiders connecting $\lambda$ to $\mu$. Composition (respectively the monoidal product) is given by vertical (respectively horizontal) juxtaposition of diagrams. These satisfy the following relations
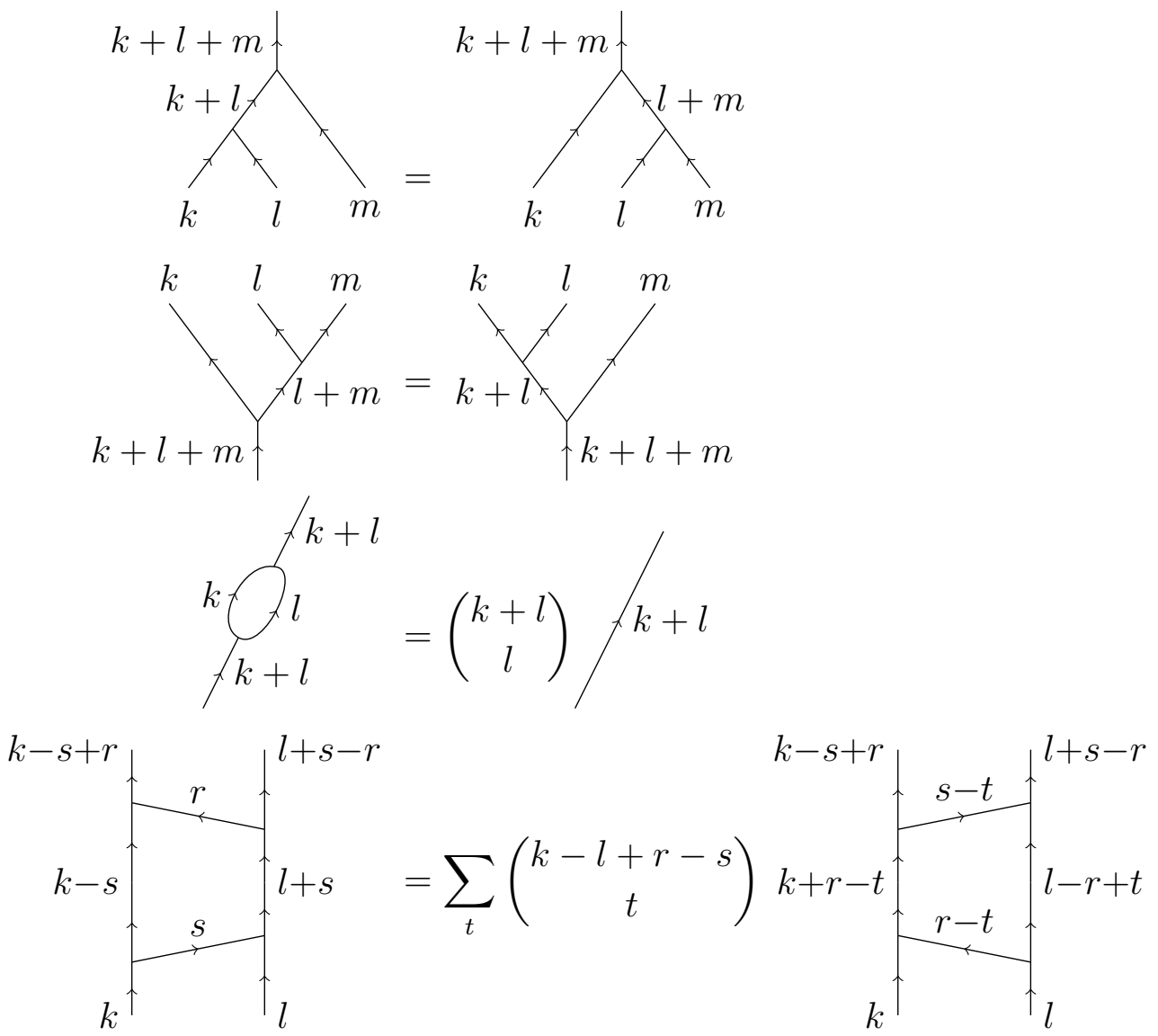

where $k, l, r, s \in \mathbb{Z}$.

As an example, let us show that the following equation holds in $\mathcal{S p}(\mathcal{P} e r m)$.

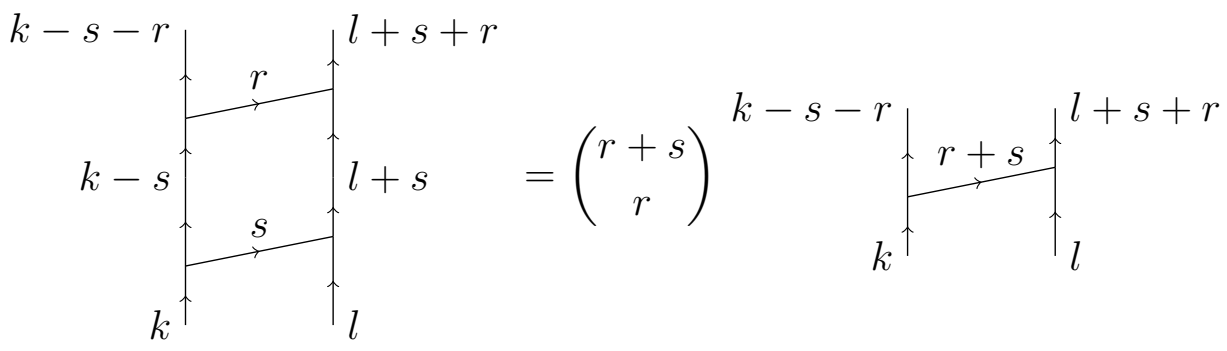


Indeed equation (3.5) follows by applying (3.2) to the left and right upright strands, then applying (3.3) to the resulting bigon.

Define the following morphisms in Perm:

$$
\begin{array}{ll}
\nabla_{k, l}: M^{k, l} \rightarrow \mathbb{C}=M^{k+l} ; \sum_{g \in G} k_{g} g \mapsto \sum_{g \in G} k_{g} & \text { for } k_{g} \in \mathbb{C} \\
\Delta_{k, l}: \mathbb{C}=M^{k+l} \rightarrow M^{k, l} ; 1 \mapsto \sum_{g \in S_{k, l} / S_{k+l}} g=\frac{1}{k ! l !} \sum_{g \in S_{k+l}} S_{k, l} g &
\end{array}
$$

Theorem 3.1.1. There is an isomorphism of $\mathbb{C}$-linear monoidal categories $\Gamma: \mathcal{S} p(\mathcal{P} e r m) \rightarrow$ Perm defined

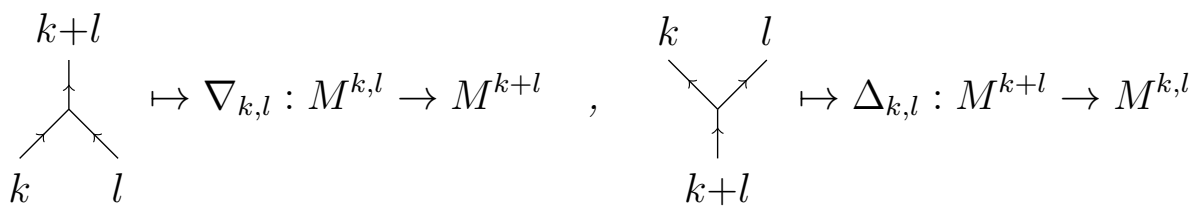

In particular, the morphisms of $\mathcal{P}$ erm are o-generated by the morphisms $\nabla_{k, l}$ and $\Delta_{k, l}$.

The proof of Theorem 3.1.1 will cover Sections 3.2, 3.3, and 3.4. In Section 3.2 we give a diagrammatic presentation of $\dot{\mathcal{U}} \mathfrak{g l}_{n}$. In Section 3.3 we apply Schur-Weyl Duality and the CKM Principle to identify the category, $\dot{\mathcal{S}}(n, d)$, of weight spaces of $\bigotimes^{d} \mathbb{C}^{n}$ with a quotient of $\dot{\mathcal{U}} \mathfrak{g l}_{n}$. In Section 3.4 we use this presentation of $\dot{\mathcal{S}}(n, d)$ to construct a presentation of $\mathcal{P}$ erm.

3.2. The category $\dot{\mathcal{U}} \mathfrak{g l}_{n}$. Fix the Cartan subalgebra, $\mathfrak{h}$, of $\mathfrak{g l}_{n}$ to be the space of diagonal matrices. We write integral weights of $\mathfrak{g l}_{n}$ by their vector coordinates with respect to the basis of $\mathfrak{h}^{*}$ dual to the standard basis $e_{11}, \ldots, e_{n n}$ of $\mathfrak{h}$. Let $\alpha_{i}$ be the sequence $(0, \ldots, 0,1,-1,0, \ldots, 0) \in \mathbb{Z}^{n}$, where the 1 is in the $i$-th position.

The $\mathbb{C}$-linear category $\dot{\mathcal{U}} \mathfrak{g l} \mathfrak{l}_{n}$ is defined:

- Objects: Sequences $\lambda=\left(\lambda_{1}, \ldots, \lambda_{n}\right) \in \mathbb{Z}^{n}$.

- Morphisms: The morphisms are generated by $E_{i}^{(r)} 1_{\lambda} \in 1_{\lambda+r \alpha_{i}} \dot{\mathcal{U}} \mathfrak{g l}_{n} 1_{\lambda}$ and $F_{i}^{(r)} 1_{\lambda} \in 1_{\lambda-r \alpha_{i}} \dot{\mathcal{U}} \mathfrak{g l}_{n} 1_{\lambda}$ for $1 \leq i \leq n-1$ and satisfy the relations:

$$
\begin{aligned}
E_{i}^{(r)} F_{i}^{(s)} 1_{\lambda}=\sum_{t}\left(\begin{array}{cr}
\lambda_{i}-\lambda_{i+1}+r-s \\
t
\end{array}\right) F_{i}^{(s-t)} E_{i}^{(r-t)} 1_{\lambda} & \\
E_{i}^{(r)} F_{j}^{(s)} 1_{\lambda}=F_{j}^{(s)} E_{i}^{(r)} 1_{\lambda}, & \text { if } i \neq j \\
E_{i} E_{j} E_{i} 1_{\lambda}=\left(E_{i}^{(2)} E_{j}+E_{j} E_{i}^{(2)}\right) 1_{\lambda}, & \text { if }|i-j|=1 \\
E_{i}^{(r)} E_{j}^{(s)} 1_{\lambda}=E_{j}^{(s)} E_{i}^{(r)} 1_{\lambda} & \text { if }|i-j|>1 \\
E_{i}^{(s)} E_{i}^{(r)} 1_{\lambda}=\left(\begin{array}{c}
r+s \\
r
\end{array}\right) E_{i}^{(r+s)} 1_{\lambda} &
\end{aligned}
$$

and likewise with $F$ and $E$ interchanged in equations (3.8), (3.9), (3.10). 
The generating morphisms $E_{i} 1_{\lambda}$ and $F_{i} 1_{\lambda}$ correspond to the Chevalley generators $E_{i}=e_{i, i+1}$ and $F_{i}=e_{i+1, i}$ for $1 \leq i \leq n-1$. The generating morphisms $E_{i}^{(r)} 1_{\lambda}$ and $F_{i}^{(r)} 1_{\lambda}$ for $r>1$ are the divided powers

$$
E_{i}^{(r)} 1_{\lambda}=\frac{1}{r !} E_{i}^{r} 1_{\lambda} \quad \text { and } \quad F_{i}^{(r)} 1_{\lambda}=\frac{1}{r !} F_{i}^{r} 1_{\lambda}
$$

We give the presentation of $\dot{\mathcal{U}} \mathfrak{g l}{ }_{n}$ in terms of these redundant generators because they are more amenable to diagrammatic calculus.

Cautis-Kamnitzer-Morrison [CKM14] identified morphisms in $\dot{\mathcal{U}} \mathfrak{g l}_{n}$ with "ladder" diagrams. We define an $n$-ladder to be an oriented plane diagram consisting of $n$ upwards oriented vertical strands labelled by integers, and (horizontal) strands connecting adjancent uprights and labeled by nonnegative integers. Following [CKM14] we identify morphisms in $\dot{\mathcal{U}} \mathfrak{g l}_{n}$ with diagrams in the following way,

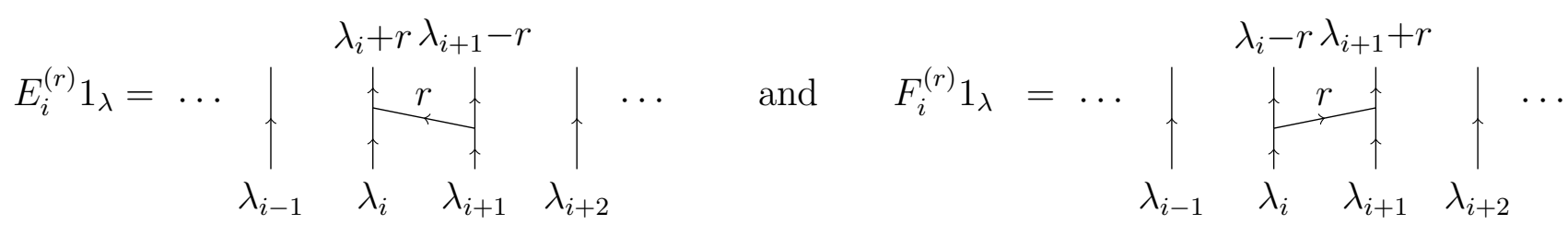

with composition of morphisms drawn by vertical juxtaposition. The relations (3.6)(3.10) simplify to invariance under any planar isotopy that preserves the upwardorientation of the strands, as well as the following relations,
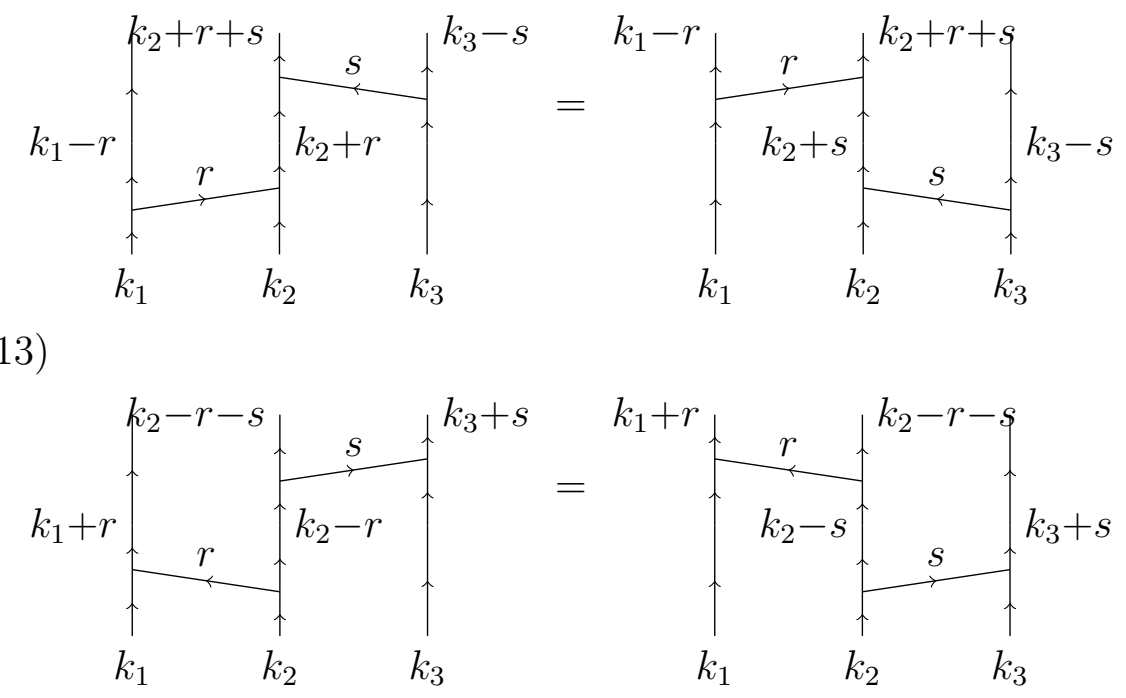

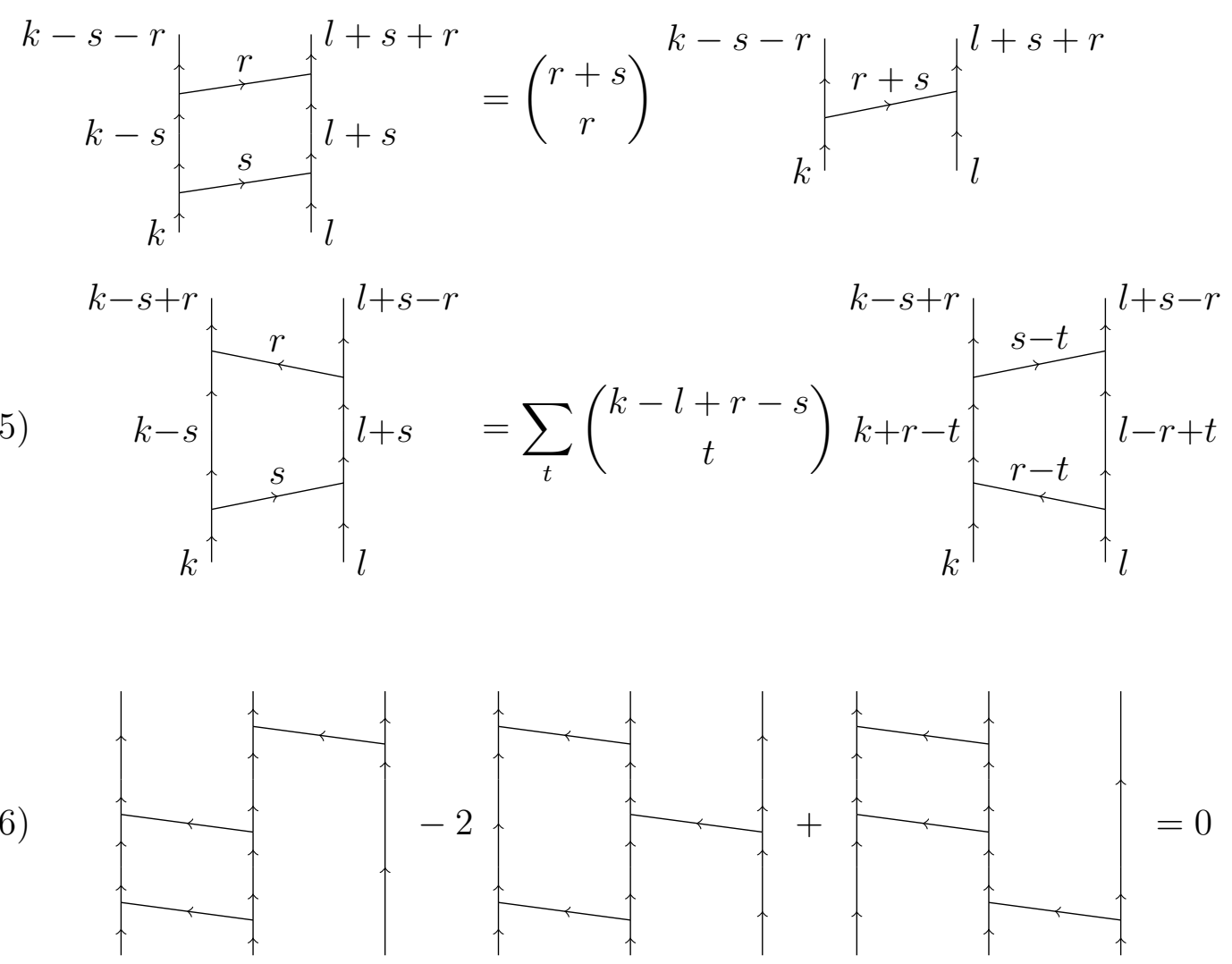

together with their mirror reflections in the $y$-axis. We interpret unlabeled horizontal strands as being labeled by 1 , and unlabeled vertical strands as being labeled by arbitrary compatible labels. These diagrams are to be interpreted as having some number of vertical strands to the left and right.

Define a $\mathfrak{g l}_{n}$-ladder to be an equivalence class of $n$-ladders, where the equivalence relation is given by equations (3.12) - (3.16) and invariance under orientationpreserving planar isotopy. Given two sequences $\lambda, \mu$ of integers we say that a $\mathfrak{g l}_{n^{-}}$ ladder connects $\lambda$ to $\mu$ if the sequence of edges along the bottom (respectively top) of the ladder diagram is $\lambda$ (respectively $\mu$ ).

By definition of $\mathfrak{g l}_{n}$-ladders we have that $\dot{\mathcal{U}} \mathfrak{g l}_{n}$ is isomorphic to the category with

- Objects: Sequences $\lambda \in \mathbb{Z}^{n}$.

- Morphisms: Morphisms from $\lambda$ to $\mu$ are $\mathbb{C}$-linear combinations of $\mathfrak{g l}_{n}$-ladders connecting $\lambda$ to $\mu$.

3.3. Schur-Weyl Duality and the CKM Principle. Let $V:=\mathbb{C}^{n}$. The weights of $V^{\otimes d}$ are elements of the set

$$
\Lambda(n, d):=\left\{\left(\lambda_{1}, \ldots, \lambda_{n}\right) \in \mathbb{Z}_{\geq 0}^{n} \mid \sum_{i} \lambda_{i}=d\right\}
$$


Let $\Lambda^{+}(n, d)$ be the set of $\mathfrak{g l}_{n}$-dominant weights of $\Lambda(n, d)$. Schur-Weyl Duality states that $V^{\otimes d}$ is a $\left(\mathfrak{g l}_{n}, \mathbb{C}\left[S_{d}\right]\right)$-bimodule with saturated multiplicity free decomposition

$$
V^{\otimes d}=\bigoplus_{\lambda \in \Lambda^{+}(n, d)} V^{\lambda} \otimes L^{\lambda}
$$

where the $V^{\lambda}$ are the irreducible $\mathfrak{g l}_{n}$-modules of highest weight $\lambda$, and the $L^{\lambda}$ are the Specht modules.

Proposition 3.3.1. Let $\dot{\mathcal{S}}(n, d)$ be the full subcategory of $\mathcal{R} e p S_{d}$ whose objects are the $\mathfrak{g l}_{n}$-weight spaces of $V^{\otimes d}$. Then $\dot{\mathcal{S}}(n, d)$ is isomorphic to the category defined

- Objects: Sequences $\lambda \in \Lambda(n, d)$.

- Morphisms: Morphisms from $\lambda$ to $\mu$ are $\mathbb{C}$-linear combinations of $\mathfrak{g l}_{n}$-ladders connecting $\lambda$ to $\mu$, and satisfying the relation

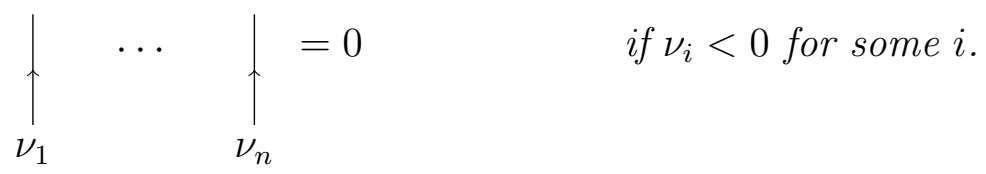

Proof. By the CKM principle $\dot{\mathcal{S}}(n, d)$ is isomorphic to $\dot{\mathcal{U}}^{V^{\otimes d}} \mathfrak{g l}_{n}$. Hence it suffices to show that (3.17) is equivalent to the relation

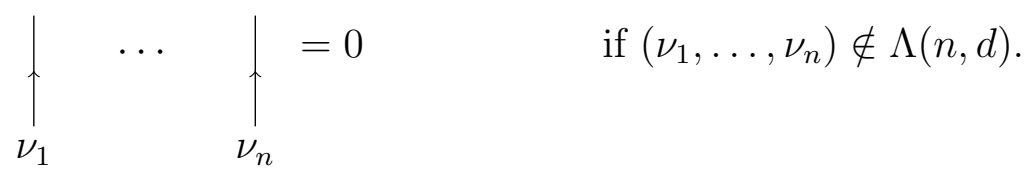

Indeed $\nu \notin \Lambda(n, d)$ if and only if either $\sum_{i} \nu_{i} \neq d$ or $\nu_{i}<0$ for some $i$. However it is impossible for a $\mathfrak{g l}_{n}$-ladder to connect a sequence $\lambda \in \Lambda(n, d)$ to a sequence $\nu \in \mathbb{Z}^{n}$ in which $\sum_{i} \nu_{i} \neq d$. The result follows.

By Proposition 3.3.1 there is a bifunctor $\cdot \| \cdot: \dot{\mathcal{S}}(n, d) \times \dot{\mathcal{S}}\left(m, d^{\prime}\right) \rightarrow \dot{\mathcal{S}}(n+m, d+$ $d^{\prime}$ ) defined on objects by $\left(\left(\mathbb{C}^{n}\right)_{\lambda}^{\otimes d},\left(\mathbb{C}^{m}\right)_{\mu}^{\otimes d^{\prime}}\right) \mapsto\left(\mathbb{C}^{m+n}\right)_{(\lambda, \mu)}^{\otimes d+d^{\prime}}$, and on morphisms by horizontal juxtaposition of ladder diagrams.

Remark. For bifunctoriality of $\cdot \| \cdot$ we need that $\mathfrak{g l}_{n}$-ladders are invariant under orientation preserving planar isotopy.

Proposition 3.3.2. The bifunctor $\| \cdot$ is equal to the circle product bifunctor $\cdot \circ \cdot=$ $\operatorname{Ind}_{S_{d} \times S_{d^{\prime}}}^{S_{d+d^{\prime}}}(\cdot \otimes \cdot): \dot{\mathcal{S}}(n, d) \times \dot{\mathcal{S}}\left(m, d^{\prime}\right) \rightarrow \dot{\mathcal{S}}\left(n+m, d+d^{\prime}\right)$.

Proof. It suffices to show that the bifunctors $\cdot \| \cdot$ and $\cdot \circ \cdot$ are equal on objects and generating morphisms. Let $\lambda \in \Lambda(n, d)$ and $\mu \in \Lambda\left(m, d^{\prime}\right)$. Write $E_{i}^{(r)} 1_{\lambda}:\left(\mathbb{C}^{n}\right)_{\lambda}^{\otimes d} \rightarrow$ $\left(\mathbb{C}^{n}\right)_{\lambda+\alpha_{i}}^{\otimes d}$ for the $S_{d^{-}}$equivariant morphism $v \mapsto \frac{1}{r !} E_{i}^{r} v$, and $1_{\lambda}$ for the identity on $\left(\mathbb{C}^{n}\right)_{\lambda}^{\otimes d}$. It suffices to show that

$$
E_{i}^{(r)} 1_{\lambda} \circ 1_{\mu}=E_{i}^{(r)} 1_{(\lambda, \mu)}:\left(\mathbb{C}^{m+n}\right)_{(\lambda, \mu)}^{\otimes d+d^{\prime}} \rightarrow\left(\mathbb{C}^{m+n}\right)_{\left(\lambda+\alpha_{i}, \mu\right)}^{\otimes d+d^{\prime}}
$$




$$
1_{\lambda} \circ E_{i}^{(r)} 1_{\mu}=E_{i+n}^{(r)} 1_{(\lambda, \mu)}:\left(\mathbb{C}^{m+n}\right)_{(\lambda, \mu)}^{\otimes d+d^{\prime}} \rightarrow\left(\mathbb{C}^{m+n}\right)_{\left(\lambda, \mu+\alpha_{i}\right)}^{\otimes d+d^{\prime}}
$$

and likewise with $F_{i}$ replacing $E_{i}$. We just show (3.18).

Let $\left\{v_{1}, \ldots, v_{n}\right\}$ and $\left\{w_{1}, \ldots w_{m}\right\}$ be the standard basis of $\mathbb{C}^{n}$ and $\mathbb{C}^{m}$ respectively. Write $\left\{v_{1}, \ldots, v_{n}, w_{1}, \ldots w_{m}\right\}$ for the standard basis of $\mathbb{C}^{m+n}$. There is a canonical inclusion of $\mathbb{C}\left[S_{d}\right] \times \mathbb{C}\left[S_{d^{\prime}}\right]$-modules:

$$
\iota:\left(\mathbb{C}^{n}\right)_{\lambda}^{\otimes d} \otimes\left(\mathbb{C}^{m}\right)_{\mu}^{\otimes d^{\prime}} \rightarrow\left(\mathbb{C}^{m+n}\right)_{(\lambda, \mu)}^{\otimes d+d^{\prime}} ; v \otimes w \mapsto v \otimes w
$$

Define the $\mathbb{C}\left[S_{d}\right] \times \mathbb{C}\left[S_{d^{\prime}}\right]$-equivariant morphism:

$$
\left(E_{i}^{(r)} 1_{\lambda}, 1_{\mu}\right):\left(\mathbb{C}^{n}\right)_{\lambda}^{\otimes d} \otimes\left(\mathbb{C}^{m}\right)_{\mu}^{\otimes d^{\prime}} \rightarrow\left(\mathbb{C}^{m+n}\right)_{\left(\lambda+\alpha_{i}, \mu\right)}^{\otimes d+d^{\prime}} ; v \otimes w \mapsto E_{i}^{(r)} 1_{\lambda}(v) \otimes w
$$

Then $E_{i}^{(r)} 1_{(\lambda, \mu)}$ is the unique $\mathbb{C}\left[S_{d+d^{\prime}}\right]$-equivariant morphism making the following diagram commute

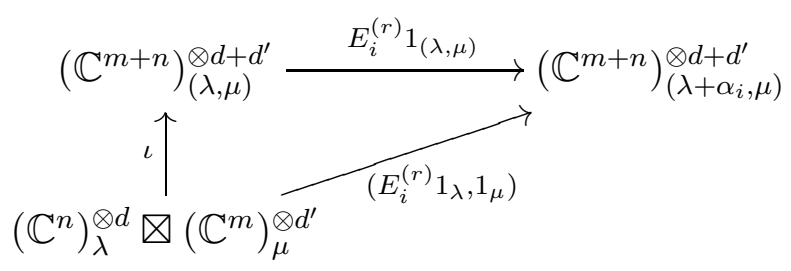

Equation (3.18) follows.

Remark. Note that the Schur Algebra $S(n, d):=\operatorname{End}_{\mathbb{C}\left[S_{d}\right]} V^{\otimes d} \cong \bigoplus_{\lambda, \mu \in \Lambda(n, d)} \operatorname{Hom}_{\dot{\mathcal{S}(n, d)}}\left(1_{\lambda}, 1_{\mu}\right)$. Hence a presentation of $S(n, d)$ is given by generators $1_{\lambda}, \lambda \in \mathbb{Z}^{n}$, and $E_{i}, F_{i}, 1 \leq i \leq n$. These satisfy relations (3.6), (3.7), (3.8), (3.9) and

$$
\begin{array}{rlrl}
1_{\lambda} & =0 & & \text { if } \lambda \notin \Lambda(n, d) \\
1_{\lambda} 1_{\mu} & =\delta_{\lambda, \mu} 1_{\lambda}, \quad \sum_{\lambda \in \mathbb{Z}^{n}} 1_{\lambda}=1 & \\
E_{i} 1_{\lambda} & =1_{\lambda+\alpha_{i}} E_{i}, \quad F_{i} 1_{\lambda}=1_{\lambda-\alpha_{i}} F_{i} &
\end{array}
$$

This is the same presentation of $S(n, d)$ derived in [Dot03].

3.4. Proof of Theorem 3.1.1. We now show that the functor $\Gamma: \mathcal{S} p(\mathcal{P} e r m) \rightarrow$ Perm defined in Theorem 3.1.1 is well defined and an isomorphism of categories. Our proof is essentially the same argument used in [CKM14] to give a diagrammatic presentation of the category of fundamental representations of $\mathfrak{s l}_{n}$.

We begin by defining the functor $\Theta_{d}^{n}: \dot{\mathcal{S}}(n, d) \rightarrow \mathcal{S} p(\mathcal{P}$ erm $)$

- Objects: An object $V_{\lambda}^{\otimes d}$ is mapped to the composition, $\kappa(\lambda) \vDash d$ obtained from $\lambda$ by deleting 0 terms.

- Morphisms: $\mathfrak{g l}_{n}$-ladders are identified with the $\mathcal{P}$ erm-spiders drawn the same way. 
To see that $\Theta_{d}^{n}$ is well defined we must check that equations (3.12)-(3.16) and (3.17) hold in $\mathcal{S} p($ Perm $)$. It is immediate that (3.17) holds in $\mathcal{S} p(\mathcal{P} e r m)$. Equations (3.12) and (3.13) hold in the category $\mathcal{S} p(\mathcal{P} e r m)$ by (3.1) and (3.2). Equations (3.14) and (3.15) are the same as equations and (3.5) and (3.4). Furthermore (3.16) holds in $\mathcal{S} p(\mathcal{P} e r m)$ - see ([CKM14], pp.8-9) for a proof.

For any sequence $\lambda \in \mathbb{Z}^{n}$, let $l(\lambda)$ denote the length of $\lambda$.

Lemma 3.4.1. The category $\dot{\mathcal{S}}(n, d)$ is equivalent to the full subcategory of $\mathcal{P}$ erm whose objects are the permutation modules $M_{d}^{\lambda}$ in which $l(\lambda) \leq n$. Moreover the resulting fully faithful functors $\Psi_{d}^{n}: \dot{\mathcal{S}}(n, d) \rightarrow$ Perm factor through $\Theta_{d}^{n}: \dot{\mathcal{S}}(n, d) \rightarrow$ $\mathcal{S} p(\mathcal{P e r m})$.

Proof. Let $\left\{v_{1}, \ldots, v_{n}\right\}$ denote the standard basis of $\mathbb{C}^{n}$. For $\lambda \in \Lambda(n, d)$, the $S_{d^{\text {-orbit }}}$ of

$$
v_{\lambda}:=\underbrace{v_{1} \otimes \cdots \otimes v_{1}}_{\lambda_{1} \text { times }} \otimes \underbrace{v_{2} \otimes \cdots \otimes v_{2}}_{\lambda_{2} \text { times }} \otimes \cdots \otimes \underbrace{v_{n} \otimes \cdots \otimes v_{n}}_{\lambda_{n} \text { times }}
$$

is a basis of the $\lambda$-weight space, $V_{\lambda}^{\otimes d}$, of $V^{\otimes d}$. By the orbit-stabiliser relation, $V_{\lambda}^{\otimes d}$ is isomorphic, in $\mathcal{R} e p S_{d}$, to the permutation module $\mathbb{C}\left[S_{\lambda} \backslash S_{d}\right]=M^{\kappa(\lambda)}$. The first result follows.

We now show that $\Psi_{d}^{n}$ factors through $\Theta_{d}^{n}$. It suffices to show that, for any $\lambda, \mu \in$ $\Lambda(n, d)$, the kernel of the linear map

$$
\operatorname{Hom}_{\dot{\mathcal{S}}(n, d)}\left(V_{\lambda}^{\otimes d}, V_{\mu}^{\otimes d}\right) \rightarrow \operatorname{Hom}_{\mathcal{S} p(\mathcal{P} e r m)}(\kappa(\lambda), \kappa(\mu))
$$

defined by $\Theta_{d}^{n}$ is contained in the kernel of the map

$$
\operatorname{Hom}_{\dot{\mathcal{S}}(n, d)}\left(V_{\lambda}^{\otimes d}, V_{\mu}^{\otimes d}\right) \rightarrow \operatorname{Hom}_{\mathcal{P} e r m}\left(M^{\kappa(\lambda)}, M^{\kappa(\mu)}\right)
$$

defined by $\Psi_{d}^{n}$. Since $\Psi_{d}^{n}$ is faithful, it suffices to show that $\Theta_{d}^{n}$ is faithful i.e. the equations (3.1) - (3.4) hold in $\dot{\mathcal{S}}(n, d)$. The only equation that is not immediate is (3.3), which is a special case of (3.14) with $l=k-s-r=0$.

Remark. Let $\phi_{\lambda}: V_{\lambda}^{\otimes d} \rightarrow M^{\kappa(\lambda)}$ be the isomorphism in $\mathcal{R} e p S_{d}$ defined $v_{\lambda} \cdot g \mapsto S_{\kappa(\lambda)} g$ for all $g \in S_{d}$. Then $\Psi_{d}^{n}: \dot{\mathcal{S}}(n, d) \rightarrow \mathcal{P}$ erm is defined explicitly

- Objects: An object $V_{\lambda}^{\otimes d}$ is mapped to $M^{\kappa(\lambda)}$.

- Morphisms: A morphism $f: V_{\lambda}^{\otimes d} \rightarrow V_{\mu}^{\otimes d}$ in $\dot{\mathcal{S}}(n, d)$ is mapped to the morphism $\phi_{\mu} f \phi_{\lambda}^{-1}: M^{\kappa(\lambda)} \rightarrow M^{\kappa(\mu)}$ in Perm.

Lemma 3.4.2. The functor $\Gamma: \mathcal{S} p(\mathcal{P}$ erm $) \rightarrow \mathcal{P}$ erm is well defined and is the unique monoidal functor making the following diagram commute for all $n, d$ :

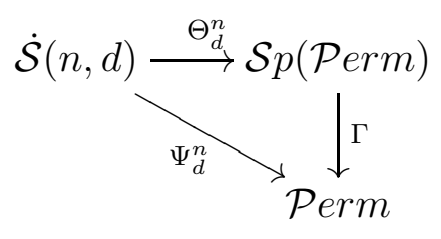


We first show that Theorem 3.1.1 follows from Lemma 3.4.2.

Each morphism in $\mathcal{P} e r m$ is of the form $\Psi_{d}^{n}(f)$ for some $n, d$. Hence (3.20) implies that $\Gamma$ is full. A special case of Theorem 5.3.1 in [CKM14] is that every Perm-spider is equal, in $\mathcal{S} p(\mathcal{P} e r m)$, to a $\mathfrak{g l}_{n}$-ladder for some $n, d$. Hence, every morphism in $\mathcal{S} p(\mathcal{P e r m})$ is of the form $\Theta_{d}^{n}(f)$ for some $n, d$. Since each $\Psi_{d}^{n}$ is faithful, this and (3.20) imply that if $\Gamma(f)=0$ then $f=0$. That is, $\Gamma$ is faithful. Finally it is clear that $\Gamma$ is bijective on objects. Hence Lemma 3.4.2 suffices to show that $\Gamma: \mathcal{S} p(\mathcal{P} e r m) \rightarrow \mathcal{P}$ erm is an isomorphism of categories.

To prove Lemma 3.4.2 we require the following sublemma.

Lemma 3.4.3. Any functor $S p(\mathcal{P} e r m) \rightarrow$ Perm making

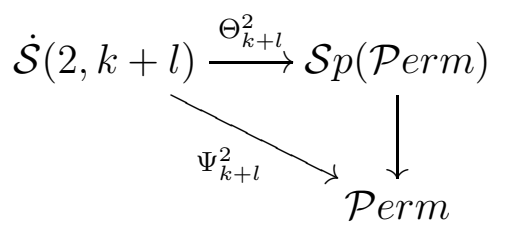

commute sends

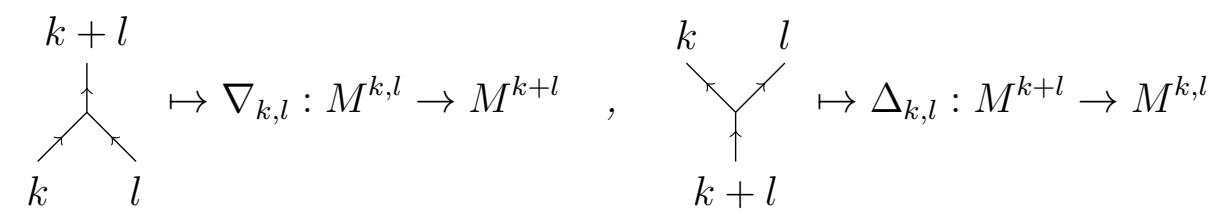

Proof. Now,

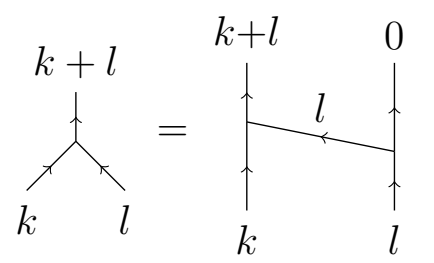

and

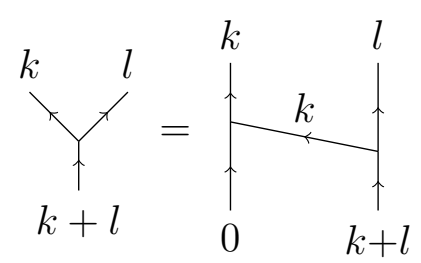

in $\mathcal{S} p(\mathcal{P}$ erm $)$. Hence it suffices to show that

$$
\begin{aligned}
\Psi_{k+l}^{2}\left(E_{1}^{(l)} 1_{k, l}\right): M^{k, l} \rightarrow M^{k+l} ; S_{k, l} g & \mapsto S_{k+l} \\
\Psi_{k+l}^{2}\left(E_{1}^{(k)} 1_{0, k+l}\right): M^{k+l} \rightarrow M^{k, l} ; S_{k+l} & \mapsto \frac{1}{k ! l !} \sum_{g \in S_{k+l}} S_{k, l} g
\end{aligned}
$$

Let $V=\mathbb{C}^{2}$. Description (3.22) holds since $E_{1}^{(l)} 1_{k, l}: V_{k, l}^{\otimes k+l} \rightarrow V_{k+l, 0}^{\otimes k+l}$ maps

$$
(\underbrace{v_{1} \otimes \cdots \otimes v_{1}}_{k \text { times }} \otimes \underbrace{v_{2} \otimes \cdots \otimes v_{2}}_{l \text { times }}) \cdot g \mapsto v_{1} \otimes \cdots \otimes v_{1}
$$

for all $g \in S_{k+l}$. Description (3.23) holds since $E_{1}^{(k)} 1_{0, k+l}: V_{0, k+l}^{\otimes k+l} \rightarrow V_{k, l}^{\otimes k+l}$ maps

$$
v_{2} \otimes \cdots \otimes v_{2} \mapsto \sum_{\substack{R \subseteq\{1, \ldots, k+l\} \\|R|=k}} v_{i_{R}(1)} \otimes \cdots \otimes v_{i_{R}(d)}, \quad \text { where } i_{R}(j)= \begin{cases}1 & \text { if } j \in R, \\ 2 & \text { otherwise }\end{cases}
$$




$$
=\frac{1}{k ! l !} \sum_{g \in S_{k+l}}(\underbrace{v_{1} \otimes \cdots \otimes v_{1}}_{k \text { times }} \otimes \underbrace{v_{2} \otimes \cdots \otimes v_{2}}_{l \text { times }}) \cdot g
$$

This completes the proof of Lemma 3.4.3.

By Lemma 3.4.1 there is a unique functor $\Theta_{d}^{n}(\dot{\mathcal{S}}(n, d)) \rightarrow \mathcal{P}$ erm making the diagram

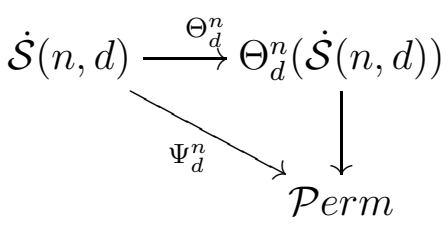

commute. By Lemma 3.4.3 and since the bifunctor $\dot{\mathcal{S}}(n, d) \times \dot{\mathcal{S}}\left(m, d^{\prime}\right) \rightarrow \dot{\mathcal{S}}(n+m, d+$ $d^{\prime}$ ) defined by horizontal juxtaposition of diagrams agrees with the circle product, this unique functor is $\left.\Gamma\right|_{\Theta_{d}^{n}(\dot{\mathcal{S}}(n, d))}: \Theta_{d}^{n}(\dot{\mathcal{S}}(n, d)) \rightarrow$ Perm. Lemma 3.4 .2 follows since every morphism in $\mathcal{S} p(\mathcal{P} e r m)$ is a morphism in $\Theta_{d}^{n}(\dot{\mathcal{S}}(n, d))$ for some $n, d$ ([CKM14], Theorem 5.3.1).

This completes the proof of Theorem 3.1.1.

\section{A Tabloid-Theoretic Description of the Generating Morphisms of $\dot{\mathcal{S}}(n, d)$}

By Proposition 3.3.1 there are morphisms

$$
\begin{aligned}
& E_{i}^{(r)} 1_{\lambda}: M^{\lambda} \rightarrow M^{\lambda+r \alpha_{i}} \\
& F_{i}^{(r)} 1_{\lambda}: M^{\lambda} \rightarrow M^{\lambda-r \alpha_{i}}
\end{aligned}
$$

that generate all $S_{d^{-}}$-equivariant morphisms between the permutation modules $M^{\lambda}$ for $\lambda \in \Lambda(n, d)$. It will be useful in Sections 5 and 6 to have an explicit description for these maps. We derive this now.

For $m \in \mathbb{N}$, define $\underline{m}:=\{1, \ldots, m\}$. The basis elements $v=v_{i_{1}} \otimes \cdots \otimes v_{i_{d}} \in V^{\otimes d}:=$ $\left(\mathbb{C}^{n}\right)^{\otimes d}$ correspond precisely to dissections, $T_{v}$, of $\underline{d}$ into $n$ subsets,

$$
T_{v}=\left\{T_{v}^{1}, \ldots, T_{v}^{n}\right\} \quad \text { where } T_{v}^{j}=\left\{k \in \underline{d} \mid i_{k}=j\right\} .
$$

If $v=v_{i_{1}} \otimes \cdots \otimes v_{i_{d}} \in V^{\otimes d}$ has weight $\lambda$, we call such a dissection a $\lambda$-tabloid (or tabloid if $\lambda$ is unspecified). We depict such a tabloid by an $n \times 1$ array of cells, indexed vertically downward, whose $j$-th cell contains the elements of $T_{v}^{j}$. For example,

$$
T_{v_{1} \otimes v_{1} \otimes v_{1} \otimes v_{2} \otimes v_{4}}=\begin{array}{|l|}
1,2,3 \\
\hline 4 \\
\hline 5 \\
\hline
\end{array}
$$

Given a tabloid $T$, write $T^{i}$ for the set of elements in the $i$-th cell of $T$. Define $Y^{\lambda}$ to be the $\mathbb{C}$-span of $\lambda$-tabloids. Now, $Y^{\lambda}$ is a right $\mathbb{C}\left[S_{d}\right]$-module with the action $T_{v} \cdot g=T_{v g}$. More precisely,

$$
(T g)^{i}=g^{-1}\left(T^{i}\right) \quad \text { for all tabloids } T \in Y^{\lambda} \text {. }
$$


By definition $Y^{\lambda}$ is isomorphic, in $\mathcal{R} e p S_{d}$, to $M^{\lambda}$. More precisely, for $\lambda \in \Lambda(n, d)$, define

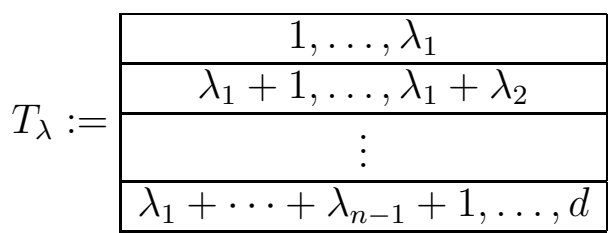

There is an isomorphism $\phi_{\lambda}: Y^{\lambda} \rightarrow M^{\lambda}$ defined $T_{\lambda} \cdot g \mapsto S_{\lambda} g$. Henceforth identify $M^{\lambda}$ with $Y^{\lambda}$ via the isomorphism $\phi_{\lambda}$.

Proposition 4.0.1. Let $R \subseteq \underline{d}, i \in \underline{n}$. For any tabloid $T$ define $c_{i, R} T$ to be the tabloid obtained from $T$ by moving every element in the set $R$ to the $i$-th cell.

The maps $E_{i}^{(r)} 1_{\lambda}: M^{\lambda} \rightarrow M^{\lambda+r \alpha_{i}}$ and $F_{i}^{(r)} 1_{\lambda}: M^{\lambda} \rightarrow M^{\lambda-r \alpha_{i}}$ are defined on tabloids by,

$$
\begin{aligned}
& E_{i}^{(r)} 1_{\lambda}: T \mapsto \sum_{R \subseteq T^{i+1},|R|=r} c_{i, R} T \\
& F_{i}^{(r)} 1_{\lambda}: T \mapsto \sum_{R \subseteq T^{i},|R|=r} c_{i+1, R} T
\end{aligned}
$$

Proof. The left action $\mathfrak{g l}_{n} \curvearrowright \bigotimes^{d} \mathbb{C}^{n}=\bigoplus_{\lambda \in \Lambda(n, d)} M^{\lambda}$ is defined on tabloids by the rule $x \cdot T_{v}=T_{x \cdot v}$ (here we interpret an expression $T_{v+w}$ as $T_{v}+T_{w}$ ). We first show that, for any tabloid $T$,

$$
e_{i j} T=\sum_{k \in T^{j}} c_{i,\{k\}} T
$$

Indeed, given $v=v_{i_{1}} \otimes \cdots \otimes v_{i_{d}}$

$$
e_{i j} v=\sum_{k \in T_{v}^{j}} v_{i_{1}} \otimes \cdots \otimes v_{i_{k-1}} \otimes v_{i} \otimes v_{i_{k+1}} \otimes \cdots \otimes v_{i_{d}}
$$

Hence,

$$
e_{i j} T_{v}=T_{e_{i j} v}=\sum_{k \in T_{v}^{j}} c_{i,\{k\}} T_{v}
$$

as required. Moreover,

$$
e_{i j}^{(r)} T=\sum_{R \subseteq T^{j},|R|=r} c_{i, R} T
$$

The result follows.

For example,

$$
F_{1} 1_{(2,1)} \cdot \frac{1,2}{3}=e_{21} \cdot T_{v_{1} \otimes v_{1} \otimes v_{2}}=T_{v_{2} \otimes v_{1} \otimes v_{2}}+T_{v_{1} \otimes v_{2} \otimes v_{2}}
$$




$$
=\begin{array}{c|}
\hline 2 \\
\hline 1,3 \\
\hline 2,3 \\
\hline 2,
\end{array}=c_{2,\{1\}} \frac{1,2}{3}+c_{2,\{2\}} \frac{1,2}{3}
$$

Remark. The maps $\nabla_{k, l}: M^{k, l} \rightarrow M^{k+l}$ and $\Delta_{k, l}: M^{k+l} \rightarrow M^{k, l}$ are defined on tabloids

$$
\begin{aligned}
\nabla_{k, l}\left(\frac{1, \ldots, k}{k+1, \ldots, k+l} \cdot g\right) & =1, \ldots, k+l \\
\Delta_{k, l}(1, \ldots, k+l & =\frac{1}{k ! l !} \sum_{g \in S_{k+l}} \frac{1, \ldots, k}{k+1, \ldots, k+l} \cdot g
\end{aligned}
$$

The circle product on permutation modules can be thought of as 'vertical juxtaposition of tabloids'. More precisely, fix $\lambda, \lambda^{\prime} \in \Lambda(n, d)$ and $\mu, \mu^{\prime} \in \Lambda\left(m, d^{\prime}\right)$. Given a $\lambda$-tabloid $T$ and $\mu$-tabloid $T^{\prime}$ define $T \circ T^{\prime}$ to be the $(\lambda, \mu)$-tabloid defined:

- For $1 \leq i \leq n,\left(T \circ T^{\prime}\right)^{i}=T^{i}$

- For $n<i \leq n+m,\left(T \circ T^{\prime}\right)^{i}=\left\{k+d \mid k \in T^{\prime i-n}\right\}$

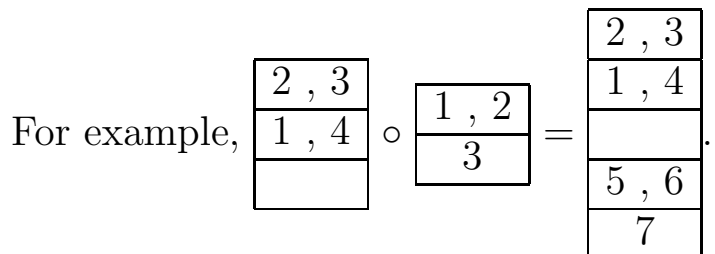

If $f: M^{\lambda} \rightarrow M^{\lambda^{\prime}}$ and $g: M^{\mu} \rightarrow M^{\mu^{\prime}}$ are $S_{d}$ and $S_{d}^{\prime}$ equivariant respectively, then $f \circ g: M^{(\lambda, \mu)} \rightarrow M^{\left(\lambda^{\prime}, \mu^{\prime}\right)}$ is the $S_{d+d^{\prime}}$-equivariant map defined on the generator $T_{(\lambda, \mu)}=T_{\lambda} \circ T_{\mu}$ :

- For $1 \leq i \leq n$,

$$
\left((f \circ g)\left(T_{\lambda, \mu}\right)\right)^{i}=\left(f\left(T_{\lambda}\right)\right)^{i}
$$

- For $n<i \leq n+m$,

$$
\left((f \circ g)\left(T_{\lambda, \mu}\right)\right)^{i}=\left\{k+d \mid k \in\left(g\left(T_{\mu}\right)\right)^{i-n}\right\}
$$

For example, $1_{M^{(1,1)}} \circ F_{1} 1_{(2,1)}: M^{(1,1,2,1)} \rightarrow M^{(1,1,1,2)}$ is defined, for $g \in S_{d}$,

$$
1_{M^{(1,1)}} \circ F_{1} 1_{(2,1)}: \begin{array}{|c|}
\hline 1 \\
\hline 2 \\
\hline 3,4 \\
\hline 5 \\
\hline 5
\end{array} \cdot g \mapsto\left(\begin{array}{|c|}
\hline 1 \\
\hline 2 \\
\hline 4 \\
\hline 3,5 \\
\hline 3
\end{array}+\begin{array}{|c|}
\hline 1 \\
\hline 2 \\
\hline 3 \\
\hline 4,5 \\
\hline
\end{array}\right.
$$

Notice from this example that $1_{M^{(1,1)}} \circ F_{1} 1_{(2,1)}=F_{3} 1_{(1,1,2,1)}$ as expected by our identification of the circle product with horizontal juxtaposition of ladder diagrams.

\section{Symmetric Monoidal Structure on Perm}

For $\lambda \in \mathbb{Z}^{n}, d \in \mathbb{Z}$, write $\lambda \vdash d$ if $\lambda$ is a partition of $d$. In this section we describe the braiding maps for the o-product in $\mathcal{P}$ erm (Theorem 5.0.1), and derive a diagrammatic presentation of the full (skeletal) subcategory, $\mathcal{P r m}$, of $\mathcal{P}$ erm whose objects are the permutation modules, $M^{\lambda}$, for $\lambda \vdash d, d \in \mathbb{N}$ (Corollary 5.1.1). 
Theorem 5.0.1. The category Perm is symmetric monoidal with braiding isomorphisms defined diagrammatically,

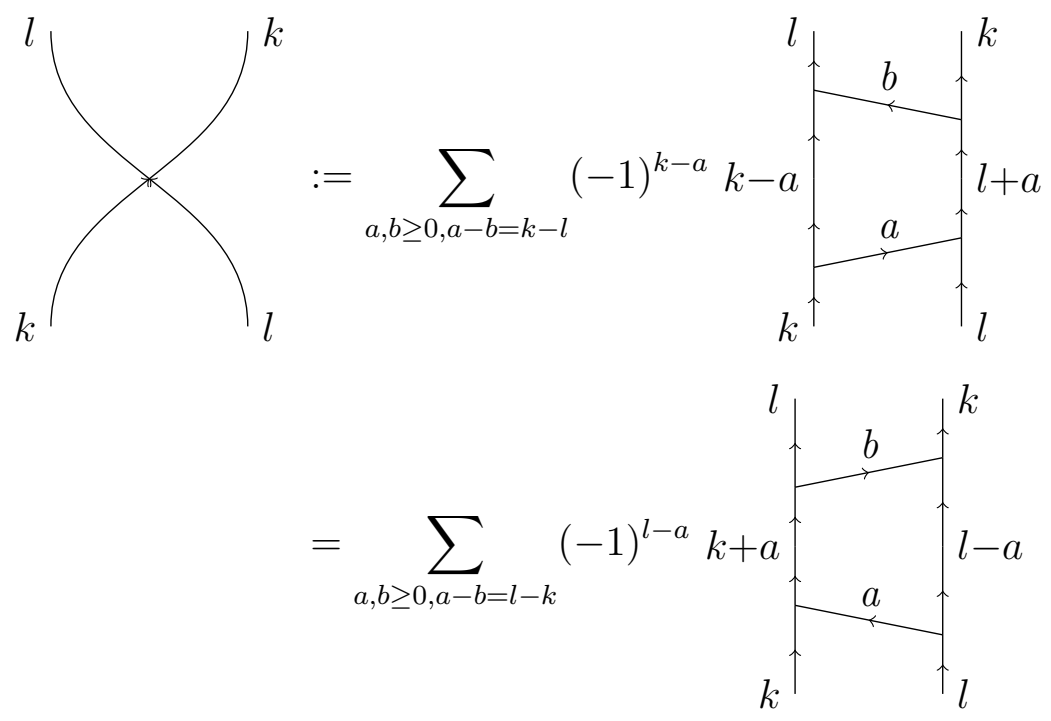

Remark. The sums in Theorem 5.0.1 are finite since we interpret strands labelled by non-positive integers as zero morphisms.

Proof. Let $B_{k, l}: M^{k, l} \rightarrow M^{l, k}$ be the isomorphism in Perm defined on $(k, l)$-tabloids by interchanging elements in the 1 st and 2 nd cells. That is,

$$
B_{k, l}(T)=c_{2, T^{1}} c_{1, T^{2}} T
$$

It is clear that the $B_{k, l}$ define braidings for $\mathcal{P}$ erm that give $\mathcal{P} e r m$ the structure of a symmetric monoidal category. Let

$$
B^{k, l}:=\sum_{a, b \geq 0, a-b=k-l}(-1)^{k-a} E_{1}^{(b)} F_{1}^{(a)} 1_{(k, l)}: M^{k, l} \rightarrow M^{l, k}
$$

We first show that $B_{k, l}=B^{k, l}$.

By Proposition 4.0.1, for any $(k, l)$-tabloid $T$,

$$
E_{1}^{(b)} F_{1}^{(a)} 1_{(k, l)}(T)=\sum_{R \subseteq T^{1},|R|=a} \sum_{S \subseteq T^{2} \cup R,|S|=b} c_{1, S} c_{2, R} T
$$

Fix $R \subsetneq T^{1}$ of size $a$, and $S \subsetneq T^{2}$ of size $b:=a-k+l$. Then

$$
c_{1, S} c_{2, R} T=c_{1, S \cup Q} c_{2, R \cup Q} T \quad \text { for all } Q \subseteq T^{1} \backslash R .
$$

In particular, $c_{1, S} c_{2, R} T$ appears $\left(\begin{array}{c}k-a \\ m\end{array}\right)=\left(\begin{array}{c}k-a \\ k-(a+m)\end{array}\right)$ times as a summand of $E_{1}^{(b+m)} F_{1}^{(a+m)}(T)$ ; once for each $Q \subseteq T^{1} \backslash R$ such that $|Q|=m$. Hence the number of times $c_{1, S} c_{2, R} T$ appears as a summand of $B^{k, l}(T)$ is

$$
\sum_{k-(a+m) \text { even }}\left(\begin{array}{c}
k-a \\
k-(a+m)
\end{array}\right)-\sum_{k-(a+m) \text { odd }}\left(\begin{array}{c}
k-a \\
k-(a+m)
\end{array}\right)=0
$$


Furthermore, $c_{1, T^{2}} c_{2, T^{1}} T$ appears exactly once in $B^{k, l}(T)$ as a summand of $E_{1}^{(l)} F_{1}^{(k)} T$. Hence,

$$
B^{k, l}(T)=c_{1, T^{2}} c_{2, T^{1}} T=B_{k, l}(T)
$$

as required.

By interchanging the roles of $T^{1}$ and $T^{2}$ in the above proof we also get that

$$
B_{k, l}=\sum_{a, b \geq 0, a-b=l-k}(-1)^{l-a} F_{1}^{(b)} E_{1}^{(a)} 1_{(k, l)}: M^{k, l} \rightarrow M^{l, k}
$$

This completes the proof of Theorem 5.0.1.

We note the following feature of the diagram calculus for $\mathcal{P} e r m$.

Proposition 5.0.2. The following relations hold in Perm:
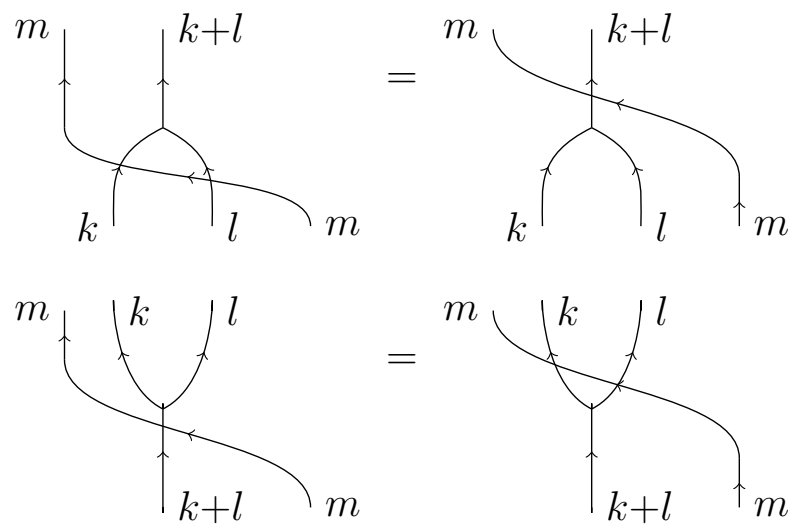

Proof. Both equations (5.1) and (5.2) can be checked by evaluating each side of the equation of tabloids. For example, both sides of equation (5.1) act on tabloids in the following way:

$$
\begin{array}{|c|}
\hline 1, \ldots, k \\
\hline k+1, \ldots, k+l \\
\hline k+l+1, \ldots, k+l+m \\
\hline
\end{array} \cdot g \mapsto \begin{gathered}
k+l+1, \ldots, k+l+m \\
\hline 1, \ldots, k+l \\
\hline
\end{gathered} g
$$

for all $g \in S_{k+l+m}$.

Remark. With a little work one may also find diagrammatic proofs of (5.1) and (5.2). We do not do this here.

5.1. A diagrammatic presentation of $\mathcal{P} r m$. Recall the symmetric group $S_{n}$ acts on $\mathbb{Z}^{n}$ by permuting entries. Given $\mu \vDash d$ and $\lambda \vdash d$, say that $\lambda$ dominates $\mu$ (write $\mu \prec \lambda)$ if $\mu$ is in the $S_{n}$ orbit of $\lambda$.

Corollary 5.1.1. The category $\mathcal{P} r m$ is isomorphic as a $\mathbb{C}$-linear monoidal category to the category with

- Objects: Partitions $\lambda \vdash d$ for some $d \in \mathbb{N}$. 
- Morphisms: Morphisms from $\lambda$ to $\mu$ are $\mathbb{C}$-linear combinations of Permspiders connecting $\lambda^{\prime}$ to $\mu^{\prime}$, where $\lambda^{\prime} \prec \lambda$ and $\mu^{\prime} \prec \mu$. These morphisms satisfy relations (3.1), (3.2), (3.3), (3.4), as well as the relation

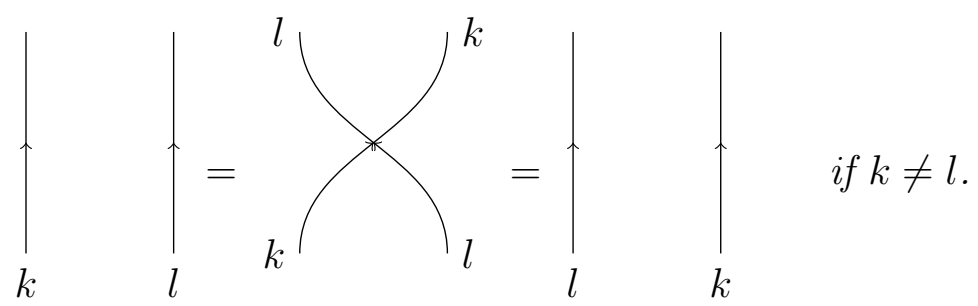

The composition of morphisms $f: \lambda \rightarrow \mu$ and $g: \mu \rightarrow \nu$ is given by vertical juxtaposition of a diagram, $\tilde{f}$, depicting $f$ above a diagram, $\tilde{g}$, depicting $g$, in which the sequence along the top of $\tilde{g}$ is the same as the sequence along the bottom of $\tilde{f}$. The monoidal product is given by horizontal juxtaposition.

Proof. Clearly two permutation modules $M^{\lambda}$ and $M^{\lambda^{\prime}}$ are isomorphic in Perm if and only if $\lambda$ and $\lambda^{\prime}$ are dominated by the same partition. Hence $\mathcal{P} r m$ is the skeleton of $\mathcal{P}$ erm. It is not hard to check that, given $\lambda^{\prime} \prec \lambda$, there is a unique isomorphism $\varphi_{\lambda^{\prime}}: M^{\lambda^{\prime}} \rightarrow M^{\lambda}$, in Perm, made up by using composition and the o-product on the braiding isomorphisms $B_{k, l}: M^{k, l} \rightarrow M^{l, k}$ where $k \neq l$. As $\mathcal{P} r m$ is the skeleton of $\mathcal{P}$ erm, $\mathcal{P r m}$ is isomorphic to the category with

- Objects: Isomorphism classes of objects in Perm. Given a partition $\lambda \vdash d$, let $[\lambda]$ denote the isomorphism class containing $M_{d}^{\lambda}$.

- Morphisms: Morphisms $[\lambda] \rightarrow[\mu]$ are equivalence classes of elements in the disjoint union

$$
\coprod_{\lambda^{\prime} \prec \lambda, \mu^{\prime} \prec \mu} \operatorname{Hom}_{\mathcal{P e r m}}\left(M^{\lambda^{\prime}}, M^{\mu^{\prime}}\right)
$$

where a morphism $f: M^{\lambda^{\prime}} \rightarrow M^{\mu^{\prime}}$ is equivalent to $\varphi_{\mu^{\prime}} f \varphi_{\lambda^{\prime}}^{-1}: M^{\lambda} \rightarrow M^{\mu}$ for all $\lambda^{\prime} \prec \lambda, \mu^{\prime} \prec \mu$.

By Theorem 3.1.1 this simplifies to the diagrammatic presentation of $\mathcal{P} r m$ given in Corollary 5.1.1.

We next prove a useful feature of the diagrammatic calculus for $\mathcal{P}$ rm.

Proposition 5.1.2. The following relations hold in Prm:

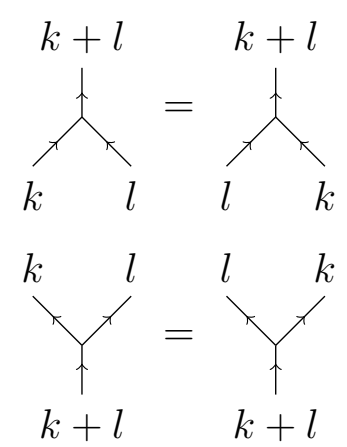


Proof. To show equation (5.4) it suffices to show that if $k \neq l$ then

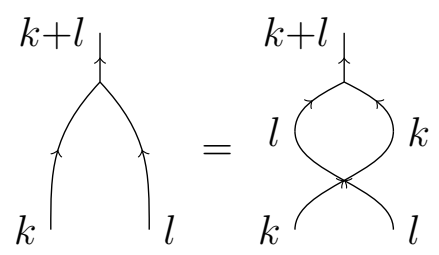

This equation can easily be verified by evaluating each side on $(k, l)$-tabloids. Equation (5.5) holds similarly.

\section{The Monoidal Structure on $\operatorname{Prm}\left(S_{d}\right)$}

Define $\operatorname{Prm}\left(S_{d}\right)$ to be the full skeletal subcategory of $\mathcal{R} e p S_{d}$ whose objects are direct sums of permutation modules $M^{\lambda}$, where $\lambda \vdash d$.

Given an object $\bigoplus_{i \in \chi} M_{i}$ in $\mathcal{P} r m\left(S_{d}\right)$, and $k \in \chi$, write $\iota_{k}: M_{i} \rightarrow \bigoplus_{i \in \chi} M_{i}$ for the canonical inclusion and $\rho_{k}: \bigoplus_{i \in \chi} M_{i} \rightarrow M_{i}$ for the canonical projection. A morphism $f: \bigoplus_{j} M_{j} \rightarrow \bigoplus_{i} M_{i}$ in $\mathcal{P} r m\left(S_{d}\right)$ can be identified with a matrix $\left(f_{i j}\right)_{i j}$ where $f_{i j}=\rho_{i} f_{\iota_{j}}: M_{j} \rightarrow M_{i}$. We slightly abuse notation by writing

$$
f=\left(f_{i j}\right)_{i j}=\sum_{i} \sum_{j} f_{i j}
$$

Given any $\lambda \in \Lambda(n, d)$ in which $\kappa(\lambda)$ is dominated by the partition $\mu \vdash d$, we may write $M^{\lambda}$ to mean the object $M^{\mu}$ in $\operatorname{Prm}\left(S_{d}\right)$. For example, $M^{(1,0,2)}$ and $M^{(2,1)}$ both denote the same object in $\operatorname{Prm}\left(S_{3}\right)$. For $\lambda \in \Lambda(n, d)$, define morphisms $E_{i} 1_{\lambda}: M^{\lambda} \rightarrow$ $M^{\lambda+\alpha_{i}}$ and $F_{i} 1_{\lambda}: M^{\lambda} \rightarrow M^{\lambda-\alpha_{i}}$ in $\mathcal{P} r m\left(S_{d}\right)$ by the diagrams

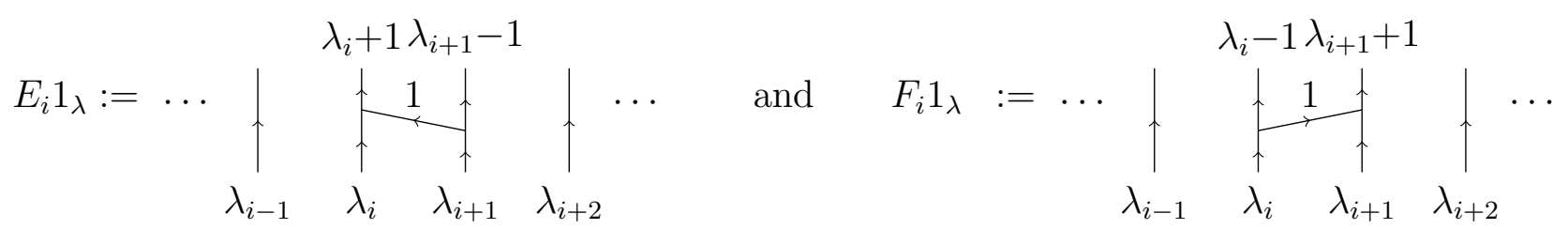

Let $\lambda, \mu \vdash d$ of length $m, n$, respectively. We define $A_{\mu}^{\lambda}$ to be the set of $m \times n$ matrices $A=\left(A_{i j}\right)_{i j}$ with entries in $\mathbb{N}$, such that $\lambda_{i}=\sum_{j} A_{i j}$ and $\mu_{j}=\sum_{i} A_{i j}$. For example

$$
A_{(2,2)}^{(3,1)}=\left\{\left[\begin{array}{ll}
2 & 1 \\
0 & 1
\end{array}\right],\left[\begin{array}{ll}
1 & 2 \\
1 & 0
\end{array}\right]\right\}, \quad A_{(3,1)}^{(3,1)}=\left\{\left[\begin{array}{ll}
3 & 0 \\
0 & 1
\end{array}\right],\left[\begin{array}{ll}
2 & 1 \\
1 & 0
\end{array}\right]\right\}
$$

Given a matrix $A \in A_{\mu}^{\lambda}$, let $M^{A}$ denote the permutation module indexed by the sequence $\left(A_{11}, A_{12}, \ldots, A_{21}, A_{22}, \ldots, A_{m n}\right)$. For $1 \leq i \leq m, 1 \leq j \leq n-1$, define morphisms $E_{i j} 1_{A}: M^{A} \rightarrow M^{A+e_{i j}-e_{i, j+1}}$ and $F_{i j} 1_{A}: M^{A} \rightarrow M^{A-e_{i j}+e_{i, j+1}}$ in $\operatorname{Prm}\left(S_{d}\right)$ 
by the diagrams,

$$
\begin{aligned}
& A_{i j}+1 \quad A_{i, j+1}-1 \\
& E_{i j} 1_{A}: M^{A} \rightarrow M^{A+e_{i j}-e_{i, j+1}}:=\uparrow_{A_{11}} \ldots
\end{aligned}
$$

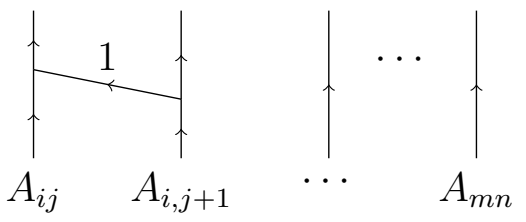

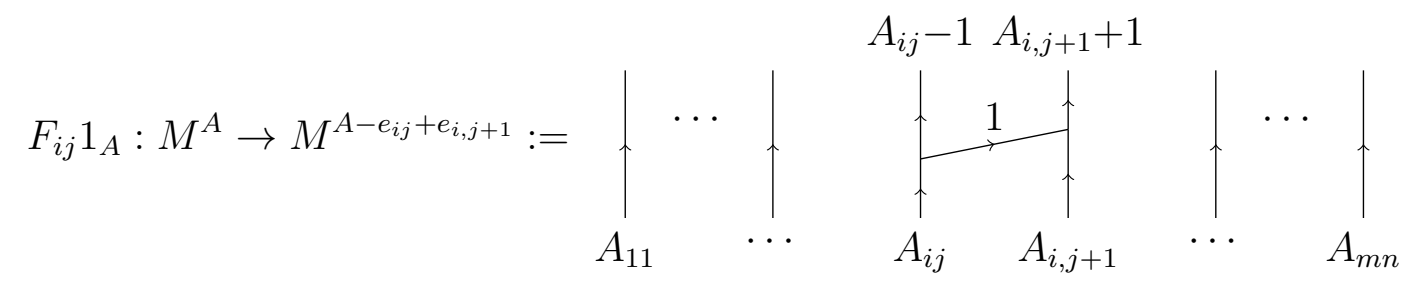

If $A \in A_{\mu}^{\lambda}$ then

(1) Either $A+\left(e_{i j}-e_{i, j+1}\right) \in A_{\mu+\alpha_{j}}^{\lambda}$ or $E_{i j} 1_{A}=0$. The latter case occurs if and only if $A_{i, j+1}=0$.

(2) Either $A-\left(e_{i j}-e_{i, j+1}\right) \in A_{\mu-\alpha_{j}}^{\lambda}$ or $F_{i j} 1_{A}=0$. The latter case occurs if and only if $A_{i j}=0$.

Hence the following theorem makes sense.

Theorem 6.0.1. The category $\operatorname{Prm}\left(S_{d}\right)$ is closed under $\otimes$-product. In particular the bifunctor $\cdot \otimes \cdot: \operatorname{Prm}\left(S_{d}\right) \times \operatorname{Prm}\left(S_{d}\right) \rightarrow \operatorname{Prm}\left(S_{d}\right)$ can be expressed,

- Objects:

$$
M^{\lambda} \otimes M^{\mu}=\bigoplus_{A \in A_{\mu}^{\lambda}} M^{A}=\bigoplus_{A \in A_{\lambda}^{\mu}} M^{A}
$$

where a matrix $A$ is regarded as the sequence $\left(A_{11}, A_{12}, \ldots, A_{21}, A_{22}, \ldots, A_{m n}\right)$.

- Morphisms: Let $\lambda, \mu \vdash d$ of length $m, n$, respectively. Then,

$$
\begin{aligned}
& E_{j} 1_{\lambda} \otimes 1_{\mu}=\sum_{A \in A_{\lambda}^{\mu}} \sum_{i=1}^{n} E_{i j} 1_{A}: \bigoplus_{A \in A_{\lambda}^{\mu}} M^{A} \rightarrow \bigoplus_{A \in A_{\lambda+\alpha_{j}}^{\mu}} M^{A} \\
& F_{j} 1_{\lambda} \otimes 1_{\mu}=\sum_{A \in A_{\lambda}^{\mu}} \sum_{i=1}^{n} F_{i j} 1_{A}: \bigoplus_{A \in A_{\lambda}^{\mu}} M^{A} \rightarrow \bigoplus_{A \in A_{\lambda-\alpha_{j}}^{\mu}} M^{A} \\
& 1_{\lambda} \otimes E_{j} 1_{\mu}=\sum_{A \in A_{\mu}^{\lambda}} \sum_{i=1}^{m} E_{i j} 1_{A}: \bigoplus_{A \in A_{\mu}^{\lambda}} M^{A} \rightarrow \bigoplus_{A \in A_{\mu+\alpha_{j}}^{\lambda}} M^{A} \\
& 1_{\lambda} \otimes F_{j} 1_{\mu}=\sum_{A \in A_{\mu}^{\lambda}} \sum_{i=1}^{m} F_{i j} 1_{A}: \bigoplus_{A \in A_{\mu}^{\lambda}} M^{A} \rightarrow \bigoplus_{A \in A_{\mu-\alpha_{j}}^{\lambda}} M^{A}
\end{aligned}
$$

Before we prove Theorem 6.0.1, let us calculate some examples. 
6.1. Examples. In all our examples $d=4$. It is easy to see that

$$
\begin{aligned}
M^{(3,1)} \otimes M^{(2,2)} & =M^{(2,1,1)} \oplus M^{(2,1,1)} \\
M^{(3,1)} \otimes M^{(3,1)} & =M^{(3,1)} \oplus M^{(2,1,1)} \\
M^{(3,1)} \otimes M^{(4)} & =M^{(3,1)}
\end{aligned}
$$

Let us show that, in matrix notation,

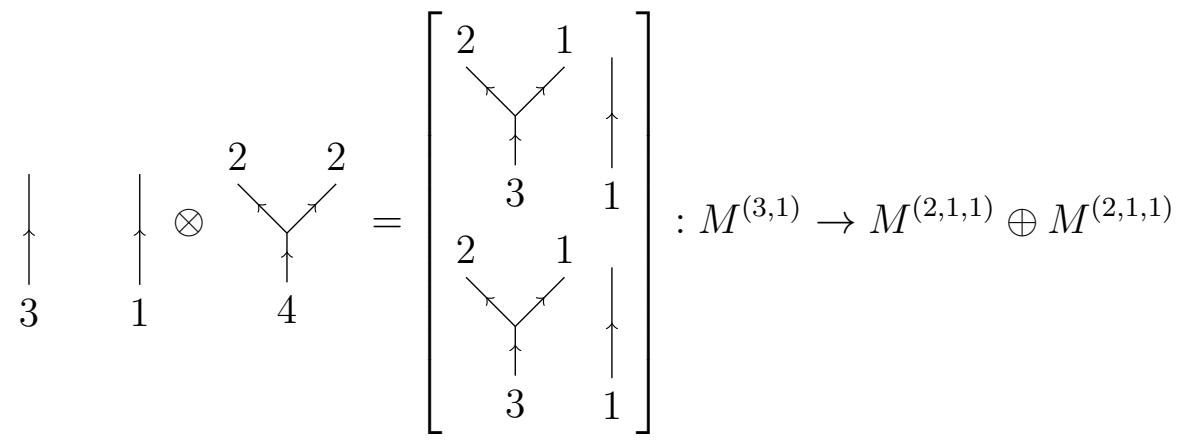

Now,

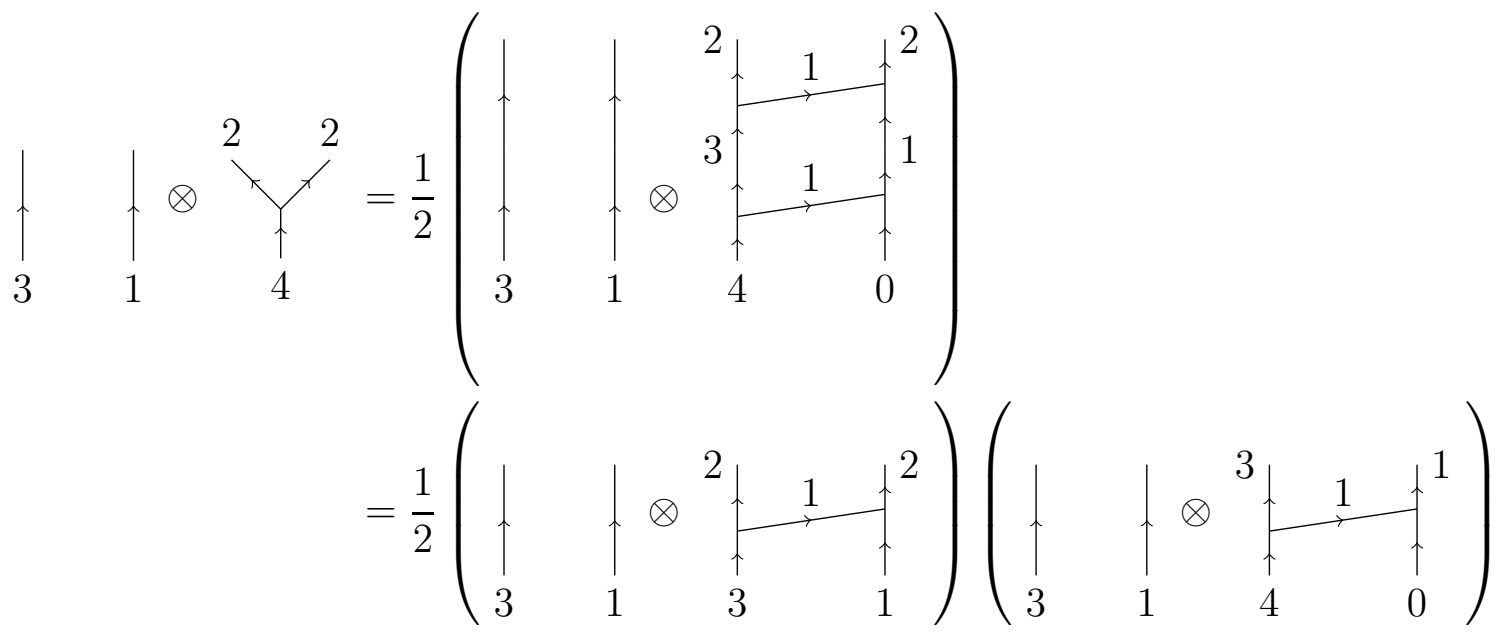


where the first equality follows from (3.5). By applying (6.3) and putting the resulting sum in matrix form we get that

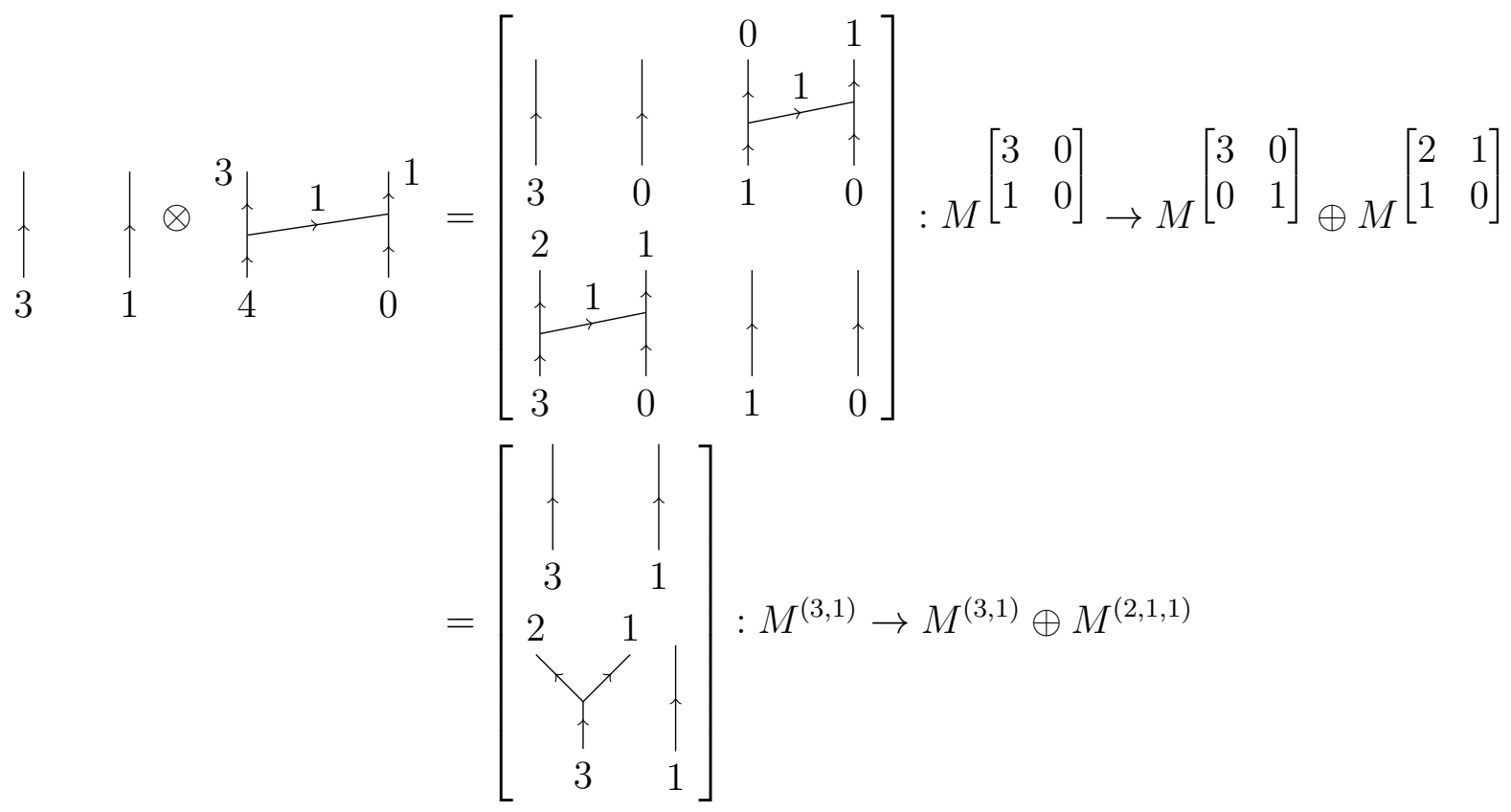

Furthermore,

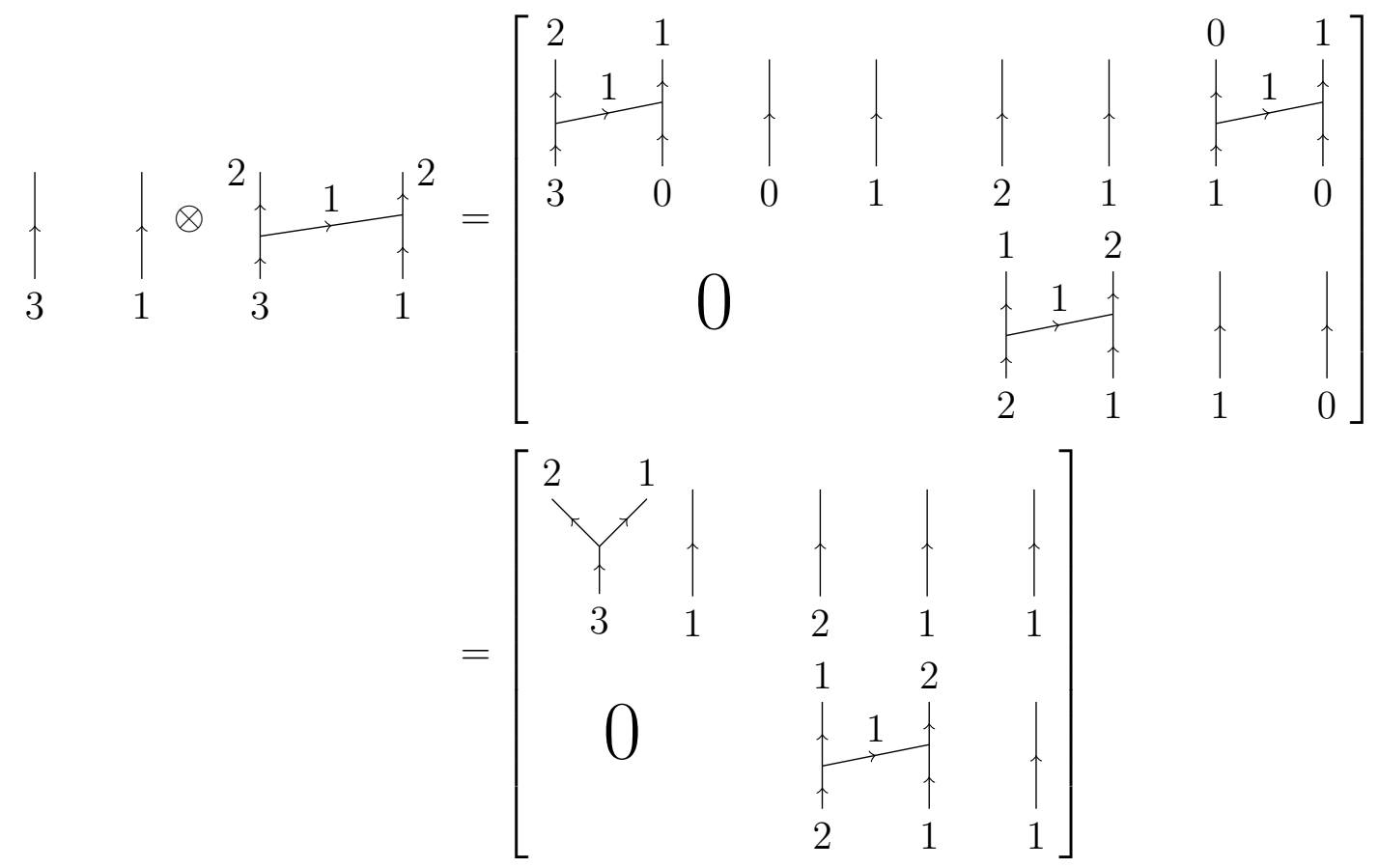


The result follows from the following matrix equation:

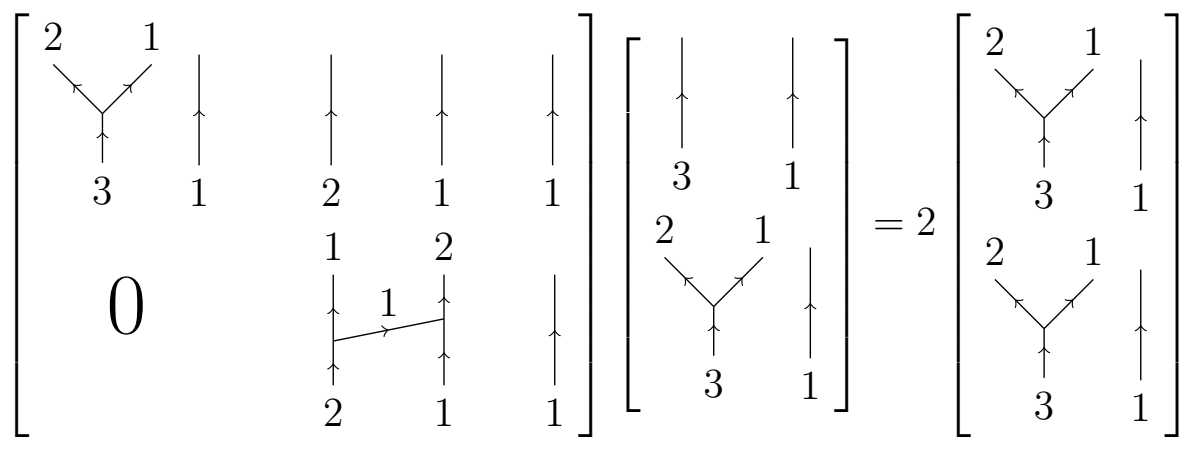

6.2. Proof of Theorem 6.0.1. Let $A$ be an $m \times n$ matrix with entries in $\mathbb{N}$, and $\sum_{i, j} A_{i j}=d$. The permutation module $M^{A}$ has a basis of dissections, $T$, of $\underline{d}$ into $m n$ subsets

$$
T=\left\{T^{11}, T^{12}, \ldots, T^{21}, T^{22}, \ldots, T^{m n}\right\}
$$

in which $A_{i j}=\left|T^{i j}\right|$. Call such a dissection an $A$-tabloid and the set $T^{i j}$ the $(i, j)$-cell of $T$.

By ([AR17], Lemma 3.1) we have the following decomposition of $S_{d}$-modules,

$$
M^{\lambda} \otimes M^{\mu}=\bigoplus_{A \in A_{\mu}^{\lambda}} M^{A}
$$

in which a tensor product $T \otimes T^{\prime} \in M^{\lambda} \otimes M^{\mu}$ is identified with the $A$-tabloid $T \otimes$ $T^{\prime} \in \bigoplus_{A \in A_{\mu}^{\lambda}} M^{A}$ defined $\left(T \otimes T^{\prime}\right)^{i j}=T^{i} \cap T^{\prime j}$. Equivalently we may index this decomposition

$$
M^{\lambda} \otimes M^{\mu}=\bigoplus_{A \in A_{\lambda}^{\mu}} M^{A}
$$

and identify a tensor product $T \otimes T^{\prime} \in M^{\lambda} \otimes M^{\mu}$ with the $A$-tabloid $T \otimes T^{\prime} \in$ $\bigoplus_{A \in A_{\lambda}^{\mu}} M^{A}$ defined $\left(T \otimes T^{\prime}\right)^{i j}=T^{j} \cap T^{\prime i}$.

We just prove equation (6.3). The proofs of the other equations are similar.

Given any $A$-tabloid $T$,

$$
E_{i j} 1_{A}(T)=\sum_{k \in T^{i, j+1}} c_{i j,\{k\}} T
$$

where $c_{i j,\{k\}} T$ is the tabloid obtained from $T$ by moving $k$ to the $(i, j)$-cell.

Fix a $\lambda$-tabloid $T$ and $\mu$-tabloid $T^{\prime}$. Define the $A$-tabloid $T \otimes T^{\prime}$ by $\left(T \otimes T^{\prime}\right)^{i j}=$ $T^{i} \cap T^{\prime j}$ as in (6.6). Note that

$$
c_{i,\{k\}} T \otimes c_{j,\{k\}} T^{\prime}=c_{i j,\{k\}}\left(T \otimes T^{\prime}\right)
$$

In particular if $k \in T^{i}$ then $T \otimes\left(c_{j,\{k\}} T^{\prime}\right)=c_{i j,\{k\}}\left(T \otimes T^{\prime}\right)$. Now,

$$
\left(1_{\lambda} \otimes E_{j} 1_{\mu}\right)\left(T \otimes T^{\prime}\right)=\sum_{k \in T^{\prime j+1}} T \otimes c_{j,\{k\}} T^{\prime}
$$




$$
\begin{aligned}
& =\sum_{i=1}^{m} \sum_{k \in T^{i} \cap T^{\prime j+1}} T \otimes c_{j,\{k\}} T^{\prime} \\
& =\sum_{i=1}^{m} \sum_{k \in\left(T \otimes T^{\prime}\right)^{i, j+1}} c_{i j,\{k\}}\left(T \otimes T^{\prime}\right) \quad \text { by }(6.7) \\
& =\sum_{i=1}^{m} E_{i j} 1_{A}\left(T \otimes T^{\prime}\right)
\end{aligned}
$$

as required.

\section{Brauer Algebras and the CKM Principle}

In this section we derive pre-Karoubi presentations of the module categories for the Brauer algebra $\mathcal{B}_{d}^{(-2 n)}$, where $2 n \geq d-1$, and the walled Brauer algebra $\mathcal{B}_{r, s}^{(n)}$, where $n \geq r+s$. We first recall the definition of the Brauer algebra.

The Brauer algebra $\mathcal{B}_{d}^{(\delta)}, \delta \in \mathbb{Z}$, has a $\mathbb{C}$-basis of $d$-diagrams. A $d$-diagram consists of $2 d$ dots arranged with $d$ dots in each of two rows, and a pairing of these dots. For example, the following is a 6-diagram,

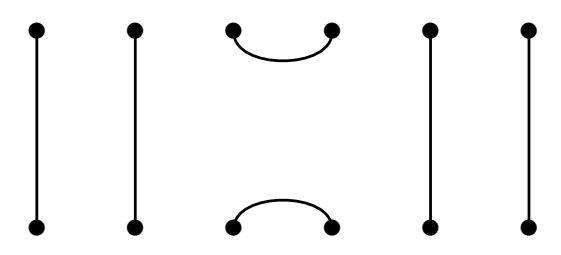

Given two $d$-diagrams $x, y$, the product $x y$ in $\mathcal{B}_{d}^{(\delta)}$ is defined as $\delta^{l} z$, where $z$ is the diagram obtained by vertical juxtaposition of $x$ above $y$ (identifying the bottom row of dots in $x$ with the top row of dots in $y$ ) and removing any resulting closed loops; and $l$ is the number of such closed loops removed.

Index the vertices on each row from left to right. Define the element $c_{i j} \in \mathcal{B}_{d}^{(\delta)}$ to be the $d$-diagram in which the $i$-th vertex on the top (respectively bottom) row is paired with the $j$-th vertex on the top (respectively bottom) row, and all other vertices are paired with the vertex directly above/below itself. For example, (7.1) is the 6-diagram $c_{34}$.

Identify $S_{d} \subset \mathcal{B}_{d}^{(\delta)}$ with the $d$-diagrams in which all vertices are paired with a vertex in a different row. More precisely, the permutation $\sigma \in S_{d}$ is identified with the $d$ diagram $\sigma \in \mathcal{B}_{d}^{(\delta)}$ defined: the $i$-th vertex on the bottom row of $\sigma \in \mathcal{B}_{d}^{(\delta)}$ is paired with the $j$-th vertex on the top row if and only if $\sigma \in S_{d}$ maps $i$ to $j$.

7.1. Schur-Weyl Duality in types C and D. We define the Lie algebra $\mathfrak{s p}_{2 n}$ and $\mathfrak{o}_{2 n}$ with respect to the skew-symmeric and symmetric forms defined respectively by the matrices $\left(\begin{array}{cc}0 & I_{n} \\ -I_{n} & 0\end{array}\right)$ and $\left(\begin{array}{cc}0 & I_{n} \\ I_{n} & 0\end{array}\right)$, where $I_{n}$ is the $n \times n$ identity matrix. We 
then get,

$$
\begin{aligned}
\mathfrak{s p}_{2 n} & =\left\{\left(\begin{array}{cc}
A & B \\
C & -A^{t}
\end{array}\right) \in \mathfrak{g l}_{2 n} \mid B=B^{t}, C=C^{t}\right\} \\
\mathfrak{o}_{2 n} & =\left\{\left(\begin{array}{cc}
A & B \\
C & -A^{t}
\end{array}\right) \in \mathfrak{g l}_{2 n} \mid B=-B^{t}, C=-C^{t}\right\}
\end{aligned}
$$

Let us take $\mathfrak{g}$ to be either $\mathfrak{s p}_{2 n}$ or $\mathfrak{o}_{2 n}$. Both $\mathfrak{s p}_{2 n}$ and $\mathfrak{o}_{2 n}$ have the Cartan subalgebra $\mathfrak{h}$ spanned by the elements $Z_{i}=e_{i i}-e_{n+i, n+i}$. Write $\varepsilon_{i} \in \mathfrak{h}^{*}$ for the element dual to $Z_{i}$. We write weights of $\mathfrak{g}$-modules by their vector coordinates with respect to the basis $\left\{\varepsilon_{i}\right\}_{i=1, \ldots, n}$ of $\mathfrak{h}^{*}$. The set of integral weights of $\mathfrak{g}$ is $\mathbb{Z}^{n}$ if $\mathfrak{g}=\mathfrak{s p}_{2 n}$ and $\mathbb{Z}^{n} \cup\left(\left(\frac{1}{2}, \ldots, \frac{1}{2}\right)+\mathbb{Z}^{n}\right)$ if $\mathfrak{g}=\mathfrak{o}_{2 n}$. The $\mathfrak{g}$-dominant weights are those weights $\left(\lambda_{1}, \ldots, \lambda_{n}\right)$ in which

$$
\begin{array}{rr}
\lambda_{1} \geq \cdots \geq \lambda_{n} \geq 0 & \text { if } \mathfrak{g}=\mathfrak{s p}_{2 n} \\
\lambda_{1} \geq \cdots \geq \lambda_{n-1} \geq\left|\lambda_{n}\right| & \text { if } \mathfrak{g}=\mathfrak{o}_{2 n}
\end{array}
$$

Let $\left\{v_{1}, \ldots, v_{n}, v_{-1}, \ldots, v_{-n}\right\}$ denote the standard basis of $\mathbb{C}^{2 n}$. Then with respect to the skew-symmetric form defined above, $v_{-i}$ is dual to $v_{i}$. Furthermore, under the natural action of $\mathfrak{s p}_{2 n}$ and $\mathfrak{o}_{2 n}$ on $\mathbb{C}^{2 n}$, the vector $v_{ \pm i}$ spans the $\pm \varepsilon_{i}$ weight space of $\mathbb{C}^{2 n}$. In either case $\mathfrak{g}=\mathfrak{s p}_{2 n}$ or $\mathfrak{g}=\mathfrak{o}_{2 n}$, the $\mathfrak{g}$-module $W=\bigotimes^{d} \mathbb{C}^{2 n}$ has the set of weights

$$
\Pi(W)=\bigcup_{j=0}^{\left\lfloor\frac{d}{2}\right\rfloor}\left\{\lambda \in \mathbb{Z}^{n}\left|\sum_{i}\right| \lambda_{i} \mid=d-2 j\right\}
$$

There is a right action of $\mathcal{B}_{d}^{(-2 n)}$ on $W$ commuting with the natural action of $\mathfrak{s p}_{2 n}$. For $i, j \in\{ \pm 1, \ldots, \pm n\}$ define

$$
\epsilon_{i j}= \begin{cases}1 & \text { if } i=-j \text { and } i>0 \\ -1 & \text { if } i=-j \text { and } i<0 \\ 0 & \text { otherwise }\end{cases}
$$

and let $\varepsilon: S_{d} \rightarrow\{ \pm 1\}$ be the sign map. The right action of $\mathcal{B}_{d}^{(-2 n)}$ on $W$ is given by,

$$
\begin{aligned}
\left(v_{i_{1}} \otimes \cdots \otimes v_{i_{d}}\right) \cdot \sigma & =\varepsilon(\sigma) v_{i_{\sigma(1)}} \otimes \cdots \otimes v_{i_{\sigma(d)}} & \text { for } \sigma \in S_{d} \subset \mathcal{B}_{d}^{(-2 n)} \\
\left(v_{i_{1}} \otimes \cdots \otimes v_{i_{d}}\right) \cdot c_{j, j+1} & =\epsilon_{i_{j}, i_{j+1}} v_{i_{1}} \otimes \cdots \otimes v_{i_{j-1}} \otimes & \\
& \left(\sum_{k=1}^{n}\left(v_{-k} \otimes v_{k}-v_{k} \otimes v_{-k}\right)\right) \otimes v_{i_{j+2}} \otimes \cdots \otimes v_{i_{n}} &
\end{aligned}
$$

The $\left(\mathfrak{s p}_{2 n}, \mathcal{B}_{d}^{(-2 n)}\right)$-bimodule $W=\bigotimes^{d} \mathbb{C}^{2 n}$ has a saturated multiplicity-free decomposition ([DGS06], Proposition 1.1.3),

$$
W=\bigoplus_{j=0}^{\left\lfloor\frac{d}{2}\right\rfloor} \bigoplus_{\lambda \in \Lambda^{+}(n, d-2 j)} V^{\lambda} \otimes \Delta^{\lambda}
$$


where $V^{\lambda}$ is the irreducible $\mathfrak{s p}_{2 n}$-module of highest weight $\lambda$, and the $\Delta^{\lambda}$ are irreducible $\mathcal{B}_{d}^{(-2 n)}$ modules. If $2 n \geq d-1$ then $\mathcal{B}_{d}^{(-2 n)}=\operatorname{End}_{\mathfrak{s p}_{2 n}} W\left[\right.$ Bro56] and so $\left\{\Delta^{\lambda}\right\}_{\lambda \in \Lambda^{+}(n, d-2 j)}$ is the complete set of irreducible $\mathcal{B}_{d}^{(-2 n)}$-modules up to isomorphism. Thus, if $2 n \geq$ $d-1$, then a pre-Karoubi presentation of $\mathcal{R} e p \mathcal{B}_{d}^{(-2 n)}$ can be obtained from the CKM Principle. We do this in Proposition 7.2.1.

There is a right action of $\mathcal{B}_{d}^{(2 n)}$ on $W$ commuting with the natural action of $\mathfrak{o}_{2 n}$. This action is defined,

$$
\begin{array}{rlr}
\left(v_{i_{1}} \otimes \cdots \otimes v_{i_{d}}\right) \cdot \sigma & =v_{i_{\sigma(1)}} \otimes \cdots \otimes v_{i_{\sigma(d)}} & \text { for } \sigma \in S_{d} \subset \mathcal{B}_{d}^{(2 n)} \\
\left(v_{i_{1}} \otimes \cdots \otimes v_{i_{d}}\right) \cdot c_{j, j+1} & =\delta_{i_{j},-i_{j+1}} v_{i_{1}} \otimes \cdots \otimes v_{i_{j-1}} \otimes & \\
& \left(\sum_{k=1}^{n}\left(v_{k} \otimes v_{-k}+v_{-k} \otimes v_{k}\right)\right) \otimes v_{i_{j+2}} \otimes \cdots \otimes v_{i_{n}} &
\end{array}
$$

([DGS06], Proposition 1.1.3) shows that the $\left(\mathfrak{o}_{2 n}, \mathcal{B}_{d}^{(2 n)}\right)$-bimodule $W$ has a saturated multiplicity-free decomposition. Furthermore [Bro56] shows that if $2 n \geq d-1$ then $\mathcal{B}_{d}^{(2 n)}=\operatorname{End}_{\mathfrak{o}_{2 n}} W$. By the CKM Principle one can directly obtain a pre-Karoubi presentation of $\mathcal{R} \operatorname{ep} \mathcal{B}_{d}^{(2 n)}$ when $2 n \geq d-1$. We do not do this here.

Remark. For all $\lambda \in \Pi(W)$, a basis for the $\lambda$-weight space, $W_{\lambda}$, of $W$ (as either an $\mathfrak{s p}_{2 n}$ or $\mathfrak{o}_{2 \mathfrak{n}}$ module) is given by the $S_{d^{\text {-orbit }}}$ of the vectors of the form $v_{i_{1}} \otimes \cdots \otimes v_{i_{r}} \otimes$ $v_{-j_{1}} \otimes \cdots \otimes v_{-j_{s}}$, where each $i_{k}>0, j_{k}>0$. In particular $W_{\lambda}$ has the following direct sum decomposition of $S_{d}$-modules,

$$
\begin{aligned}
W_{\lambda} \cong & \bigoplus \bigoplus_{\substack {(r, s) \models d \\
\begin{subarray}{c}{\mu \in \Lambda(n, r) \\
\nu \in \Lambda(n, s) \\
\lambda=\mu-\nu{ ( r , s ) \models d \\
\begin{subarray} { c } { \mu \in \Lambda ( n , r ) \\
\nu \in \Lambda ( n , s ) \\
\lambda = \mu - \nu } }\end{subarray}}\left(M^{\mu} \otimes M^{\nu}\right) \otimes_{\mathbb{C}\left[S_{r} \times S_{s}\right]} \mathbb{C}\left[S_{d}\right] \\
& \bigoplus_{(r, s) \models d} \bigoplus_{\substack{\mu \in \Lambda(n, r) \\
\nu \in \Lambda(n, s) \\
\lambda=\mu-\nu}} M^{(\mu, \nu)}
\end{aligned}
$$

This agrees with the weight space decomposition of $\bigotimes^{d} \mathbb{C}^{2 n}$ given in [Dot12].

7.2. A pre-Karoubi presentation of $\mathcal{R} \operatorname{ep} \mathcal{B}_{d}^{(-2 n)}$. Our next goal is to derive a diagrammatic pre-Karoubi presentation for $\mathcal{R e p}_{\mathcal{B}}^{(-2 n)}$ where $2 n \geq d-1$. For this we need a diagrammatic presentation of $\dot{\mathcal{U}} \mathfrak{s p}_{2 n}$.

Define elements of $\mathfrak{s p}_{2 n}$,

$$
\begin{array}{rll}
E_{i}=e_{i, i+1}-e_{n+i+1, n+i} & \text { and } & F_{i}=e_{i+1, i}-e_{n+i, n+i+1} \\
X_{j}=e_{j, n+j} & \text { and } & Y_{j}=e_{n+j, j} \\
Z_{j}=e_{j j}-e_{n+j, n+j} & \text { and } & H_{i}=Z_{i}-Z_{i+1}
\end{array}
$$

where $1 \leq i \leq n-1$ and $1 \leq j \leq n$. The standard Chevalley generators of $\mathfrak{s p}_{2 n}$ are

$$
\left\{E_{i}, F_{i}, H_{i}\right\}_{i=1, \ldots, n-1} \cup\left\{X_{n}, Y_{n}, Z_{n}\right\}
$$


corresponding to the standard base $\left\{\varepsilon_{1}-\varepsilon_{2}, \ldots, \varepsilon_{n-1}-\varepsilon_{n}, 2 \varepsilon_{n}\right\}$. The elements $X_{i}$, $Y_{i}$, for $i<n$ can be defined in terms of these generators as

$$
X_{i}=\frac{1}{2}\left[E_{i},\left[E_{i}, X_{i+1}\right]\right] \quad \text { and } \quad Y_{i}=\frac{1}{2}\left[F_{i},\left[F_{i}, Y_{i+1}\right]\right]
$$

The category $\dot{\mathcal{U}}_{\mathfrak{s p}}$ has presentation,

- Objects: Sequences in $\mathbb{Z}^{n}$.

- Morphisms: Write $\varepsilon_{i}$ for the sequence in $\mathbb{Z}^{n}$ with a 1 in the $i$-th position and 0 's elsewhere. The morphisms are generated by $E_{i}^{(r)} 1_{\lambda} \in 1_{\lambda+r \alpha_{i}} \dot{\mathcal{U}}_{\mathfrak{s p}_{2 n}} 1_{\lambda}$ and $F_{i}^{(r)} 1_{\lambda} \in 1_{\lambda-r \alpha_{i}} \dot{\mathcal{U}}_{\mathfrak{s p}}{ }_{2 n} 1_{\lambda}$ for $1 \leq i \leq n-1$, and $X_{j} 1_{\lambda} \in 1_{\lambda+2 \varepsilon_{j}} \dot{\mathcal{U}}_{\mathfrak{s p}} \mathfrak{p}_{2 n} 1_{\lambda}$ and $Y_{j} 1_{\lambda} \in 1_{\lambda-2 \varepsilon_{j}} \dot{\mathcal{U}}_{\mathfrak{s p}}{ }_{2 n} 1_{\lambda}$ for $1 \leq j \leq n$. These satisfy relations (3.6), (3.7), (3.8), (3.9), (3.10) as well as,

$$
\begin{array}{rlr}
X_{j} Y_{j} 1_{\lambda} & =Y_{j} X_{j} 1_{\lambda}+\lambda_{j} 1_{\lambda} & \\
\left(X_{i}+E_{i} X_{i+1} E_{i}\right) 1_{\lambda} & =\left(E_{i}^{(2)} X_{i+1}+X_{i+1} E_{i}^{(2)}\right) 1_{\lambda} & \\
2 X_{i+1} E_{i} X_{i+1} 1_{\lambda} & =\left(X_{i+1}^{2} E_{i}+E_{i} X_{i+1}^{2}\right) 1_{\lambda} & \\
E_{i} X_{i} 1_{\lambda} & =X_{i} E_{i} 1_{\lambda} & \\
E_{i} Y_{j} 1_{\lambda} & =Y_{j} E_{i} 1_{\lambda} & \text { if } i \neq j \\
E_{i} X_{j} 1_{\lambda} & =X_{j} E_{i} 1_{\lambda} & \text { if }|i-j|>1 \\
X_{i} Y_{j} 1_{\lambda} & =Y_{j} X_{i} 1_{\lambda} & \text { if } i \neq j
\end{array}
$$

together with the equations formed by interchanging each $E_{i}$ with $F_{i}$ and each $X_{j}$ with $Y_{j}$.

Indeed equation (7.4) corresponds to the definitions of the redundant generators in (7.2). The standard Cartan matrix for $\mathfrak{s p}_{2 n}$ is

$$
\left(\begin{array}{ccccc}
2 & -1 & 0 & \cdots & 0 \\
-1 & 2 & -1 & \ddots & \vdots \\
0 & \ddots & \ddots & -1 & 0 \\
\vdots & \ddots & -1 & 2 & -1 \\
0 & \cdots & 0 & -2 & 2
\end{array}\right)
$$

The Serre relation $\operatorname{ad}^{2}\left(X_{n}\right) E_{n-1} 1_{\lambda}=0$ is a special case of (7.5). The Serre relation $\operatorname{ad}^{3}\left(E_{n-1}\right) X_{n} 1_{\lambda}=0$ follows from (7.6) and (7.4). It is not hard to check that each relation (7.3)-(7.9) must hold in $\dot{\mathcal{U}}_{\mathfrak{s p}}$ and each Serre relation, (1.1) - (1.3), defining $\dot{\mathcal{U}}_{\mathfrak{s p}_{2 n}}$ follows from the relations (3.6) - (3.10), (7.3)-(7.9). Hence this is indeed a presentation of $\dot{\mathcal{U}}_{\mathfrak{s p}_{2 n}}$.

We can identify morphisms in $\dot{\mathcal{U}}_{\mathfrak{s p}}{ }_{2 n}$ as web diagrams in the following way. Define an $n$-ladder with bells to be a diagram formed by vertical stacking of $n$-ladders 
and the following diagrams consisting of $n$ vertical strands labelled by integers,

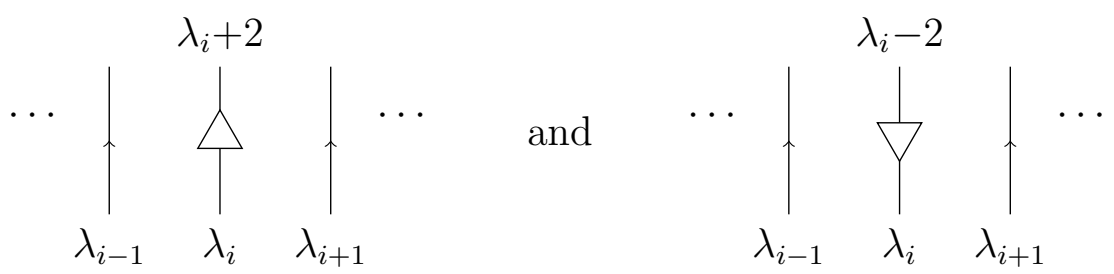

We call the above bivalent vertices, bells and cobells respectively. We identify the morphisms $E_{i}^{(r)} 1_{\lambda}$ and $F_{i}^{(r)} 1_{\lambda}$ in $\dot{\mathcal{U}} \mathfrak{s p}_{2 n}$ with $n$-ladders as in (3.11), and identify

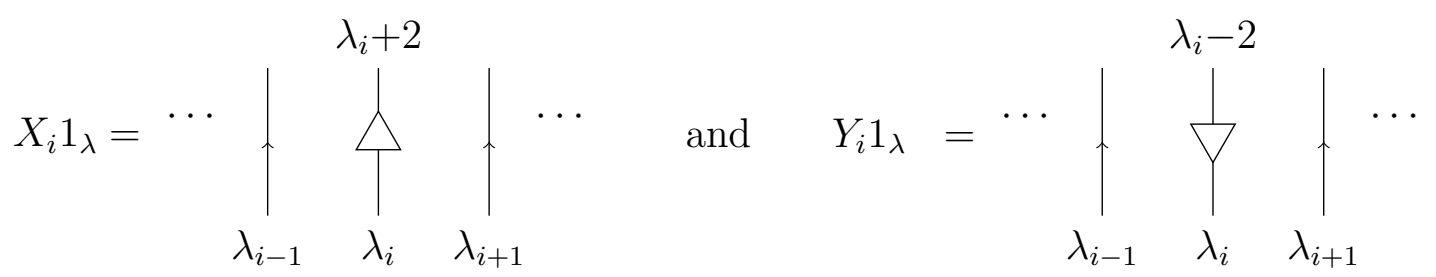

It is not hard to show that $\dot{\mathcal{U}}_{\mathfrak{s p}}{ }_{2 n}$ has presentation,

- Objects: Sequences in $\mathbb{Z}^{n}$.

- Morphisms: Morphisms from $\lambda$ to $\mu$ are $\mathbb{C}$-linear combinations of $n$-ladders with bells satisfying relations (3.12), (3.13), (3.14), (3.15), (3.16), invariance under upwards-orientation preserving planar isotopy, as well as the following relations,
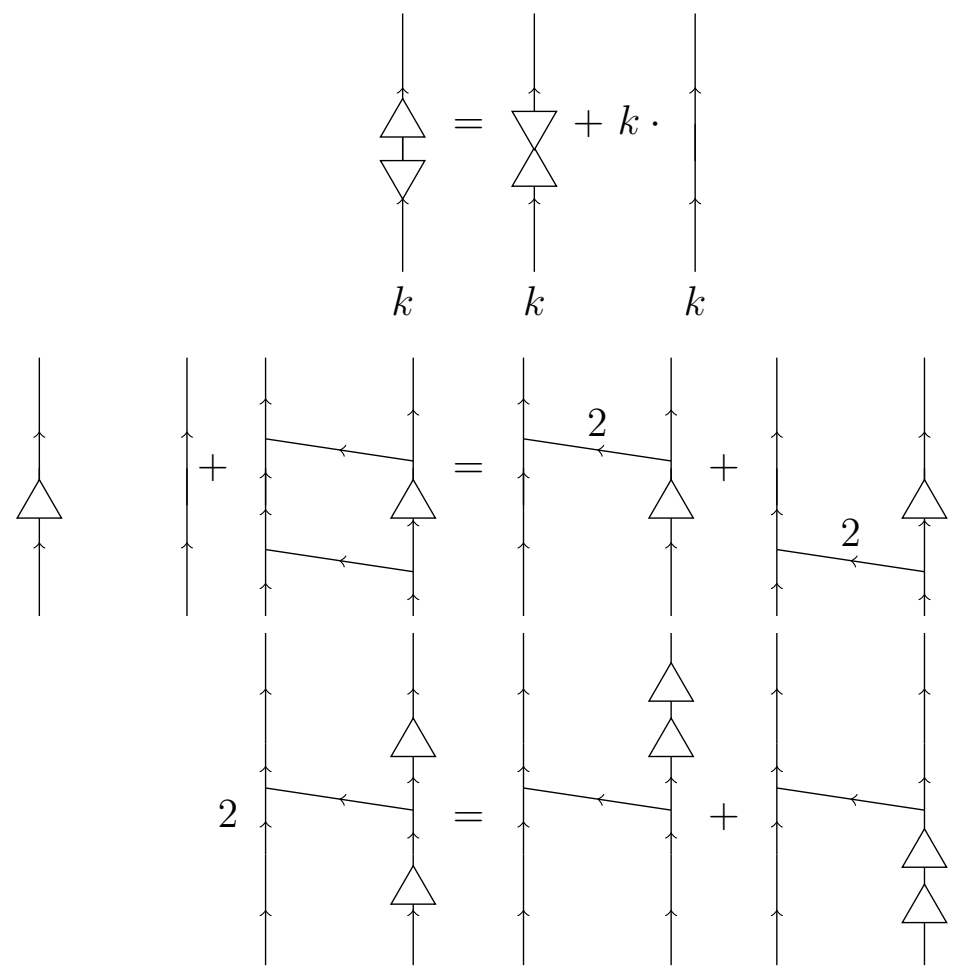


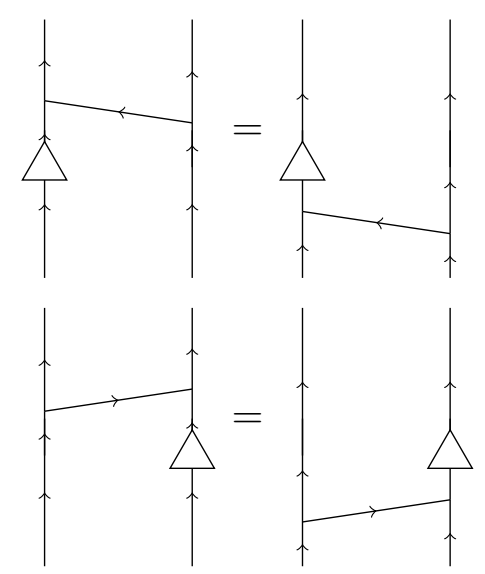

together with the relations formed by reversing the orientation of horizontal strands and interchanging bells and cobells. We interpret unlabeled horizontal strands as being labeled by 1 , and unlabeled vertical strands as being labeled by arbitrary compatible labels. These diagrams are to be interpreted as having some number of vertical strands to the left and right.

We call diagrams depicting morphisms in this presentation of $\dot{\mathcal{U}}_{\mathfrak{s p}}, \mathfrak{s p}_{2 n}$-ladders.

Remark. For $\mathfrak{s p}_{2 n}$-ladders to be invariant under planar isotopy it remains to check that the relation

$$
X_{i} X_{j} 1_{\lambda}=X_{j} X_{i} 1_{\lambda} \quad \text { if } i \neq j
$$

holds in $\dot{\mathcal{U}}_{\mathfrak{s}} \mathfrak{p}_{2 n}$. Indeed this relation can be derived from the other relations. Alternatively, this must hold in $\dot{\mathcal{U}}_{\mathfrak{s p}}$ since $\left[X_{i}, X_{j}\right]=0$ in $\mathfrak{s p}_{2 n}$.

Remark. A different diagrammatic presentation of $\dot{\mathcal{U}} \mathfrak{s p}_{2 n}$ can be obtained by choosing a different base for the weight lattice of $\mathfrak{s p}_{2 n}$ and playing this same game. Diagrammatic presentations of $\dot{\mathcal{U}} \mathfrak{o}_{2 n}$ can be also obtained in this way - cf. [ST17]. We do not do this here.

The following proposition follows by the CKM principle.

Proposition 7.2.1. Let $\dot{\mathcal{S}}_{C}(n, d)$ be the full subcategory of $\mathcal{R}$ ep $\mathcal{B}_{d}^{(-2 n)}$ whose objects are the weight spaces of $\bigotimes^{d} \mathbb{C}^{2 n}$. Then $\dot{\mathcal{S}}_{C}(n, d)$ is isomorphic to the category defined

- Objects: Sequences $\lambda \in \mathbb{Z}^{n}$ in which $\sum_{i}\left|\lambda_{i}\right|=d-2 j$ for some $0 \leq j \leq\left\lfloor\frac{d}{2}\right\rfloor$.

- Morphisms: Morphisms from $\lambda$ to $\mu$ are $\mathbb{C}$-linear combinations of $\mathfrak{s p}_{2 n}$-ladders connecting $\lambda$ to $\mu$, and satisfying the relation

$$
\uparrow_{\nu_{1}} \quad \uparrow_{\nu_{n}}=0 \quad \text { if } \sum_{i}\left|\nu_{i}\right| \neq d-2 j \text { for any } 0 \leq j \leq\left\lfloor\frac{d}{2}\right\rfloor
$$

Moreover, if $2 n \geq d-1$ then $\dot{\mathcal{S}}_{C}(n, d)$ is a pre-Karoubi subcategory of $\mathcal{R}$ ep $\mathcal{B}_{d}^{(-2 n)}$.

There is a bifunctor $\| \cdot: \dot{\mathcal{S}}_{C}(n, d) \times \dot{\mathcal{S}}_{C}\left(m, d^{\prime}\right) \rightarrow \dot{\mathcal{S}}_{C}\left(n+m, d+d^{\prime}\right)$ defined by horizontal juxtapostion of $\mathfrak{s p}_{2 n}$-ladders. 
Proposition 7.2.2. The bifunctor $\cdot \| \cdot$ is equal to the bifunctor

$$
\operatorname{Ind}_{\mathcal{B}_{d}^{(-2 n)} \times \mathcal{B}_{d^{\prime}}^{(-2 m)}}^{\mathcal{B}_{d+d^{\prime}}^{(-2 n-2 m)}}(\cdot \otimes \cdot): \dot{\mathcal{S}}_{C}(n, d) \times \dot{\mathcal{S}}_{C}\left(m, d^{\prime}\right) \rightarrow \dot{\mathcal{S}}_{C}\left(n+m, d+d^{\prime}\right) .
$$

Proof. This can be proven in the same way as Proposition 3.3.2.

7.3. Mixed Schur-Weyl Duality and the CKM principle. Consider the $\mathfrak{g l}_{n^{-}}$ module

$$
V^{r, s}:=\bigotimes^{r} \mathbb{C}^{n} \otimes \bigotimes^{s} \mathbb{C}^{* n}
$$

Benkart et. al. [BCHLLS94] described the commutant of $V^{r, s}$, for $n \geq r+s$, to be the walled Brauer algebra $\mathcal{B}_{r, s}^{(n)}$. This is the subalgebra of $\mathcal{B}_{r+s}^{(n)}$ spanned by those Brauer diagrams in which

(1) All horizontal edges pair one of the $r$ leftmost vertices with one of the $s$ rightmost vertices.

(2) No vertical edges pair one of the $r$ leftmost vertices with one of the $s$ rightmost vertices.

Write $\left\{v_{-1}, \ldots, v_{-n}\right\}$ for the standard basis of $\mathbb{C}^{* n}$ dual to the standard basis $\left\{v_{1}, \ldots, v_{n}\right\}$ of $\mathbb{C}^{n}$. The right action of $\mathcal{B}_{r, s}^{(n)}$ on $V^{r, s}$ is defined in the same way as the right action of $\mathcal{B}_{d}^{(2 n)}$ on $\bigotimes^{d} \mathbb{C}^{2 n}$.

Use square brackets for pairs of sequences. Define the set

$$
\Pi(r, s):=\{[\mu, \nu] \mid \mu \vdash r, \nu \vdash s, l(\mu)+l(\nu) \leq n\}
$$

[BCHLLS94] showed that $V^{r, s}$ has a multiplicity free decomposition

$$
V^{r, s}=\bigoplus_{j=0}^{\min \{r, s\}} \bigoplus_{[\mu, \nu] \in \Pi(r-j, s-j)} V^{[\mu, \nu]} \otimes \Delta^{[\mu, \nu]}
$$

where the $\Delta^{[\mu, \nu]}$ are irreducible $\mathcal{B}_{r, s}^{(n)}$ modules and $V^{[\mu, \nu]}$ is the irreducible $\mathfrak{g l}_{n}$-module with highest weight $\left(\mu_{1}, \ldots, \mu_{l(\mu)}, 0, \ldots, 0,-\nu_{l(\nu)}, \ldots,-\nu_{1}\right) \in \mathbb{Z}^{n}$. It is not hard to check that $V^{r, s}$ has weights

$$
\Pi\left(V^{r, s}\right)=\bigcup_{j=0}^{\min \{r, s\}}\left\{\lambda \in \mathbb{Z}^{n}\left|\sum_{i}\right| \lambda_{i} \mid=r+s-2 j, \sum_{i} \lambda_{i}=r-s\right\}
$$

and that $V^{r, s}$ is saturated. Furthermore [BCHLLS94] showed that if $n \geq r+s$ then $\mathcal{B}_{r, s}^{(n)}=\operatorname{End}_{\mathfrak{g l}_{n}} V^{r, s}$. Hence we have the following proposition.

Proposition 7.3.1. Let $\dot{\mathcal{S}}(n ; r, s)$ be the full subcategory of $\mathcal{R}$ ep $\mathcal{B}_{r, s}^{(n)}$ whose objects are the weight spaces of $V^{r, s}$. Then $\dot{\mathcal{S}}(n ; r, s)$ is isomorphic to the category defined

- Objects: Sequences $\lambda \in \Pi\left(V^{r, s}\right)$. 
- Morphisms: Morphisms from $\lambda$ to $\mu$ are $\mathbb{C}$-linear combinations of $\mathfrak{g l}_{n}$-ladders connecting $\lambda$ to $\mu$, and satisfying the relation

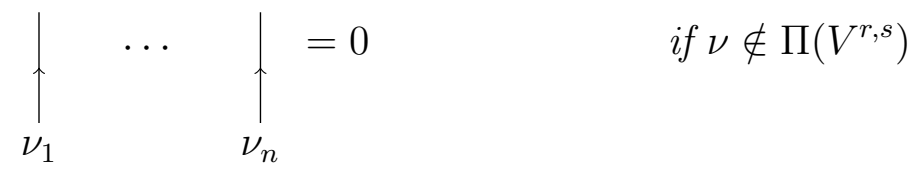

Moreover, if $n \geq r+s$ then $\dot{\mathcal{S}}(n ; r, s)$ is a pre-Karoubi subcategory of $\mathcal{R}$ ep $\mathcal{B}_{r, s}^{(n)}$.

By giving a proof similar to that of Proposition 3.3.2 we get the following result.

Proposition 7.3.2. The bifunctor $\dot{\mathcal{S}}(n ; r, s) \times \dot{\mathcal{S}}\left(m ; r^{\prime}, s^{\prime}\right) \rightarrow \dot{\mathcal{S}}\left(n+m ; r+r^{\prime}, s+s^{\prime}\right)$ defined by horizontal juxtaposition of $\mathfrak{g l}_{n}$-ladders is equal to the bifunctor

$$
\left.\operatorname{Ind}_{\mathcal{B}_{r, s}^{(n)} \times \mathcal{B}_{r^{\prime}, s^{\prime}}^{(m)}} \underset{\mathcal{B}_{r+r^{\prime}, s+s^{\prime}}^{(m+n)}}{(m)} \cdot \otimes \cdot\right): \dot{\mathcal{S}}(n ; r, s) \times \dot{\mathcal{S}}\left(m ; r^{\prime}, s^{\prime}\right) \rightarrow \dot{\mathcal{S}}\left(n+m ; r+r^{\prime}, s+s^{\prime}\right) .
$$

Remark. The $\lambda$-weight space, $V_{\lambda}^{r, s}$, of $V^{r, s}$ has the following direct sum decomposition of $\mathbb{C}\left[S_{r}\right] \times \mathbb{C}\left[S_{s}\right]$-modules,

$$
V_{\lambda}^{r, s}=\bigoplus_{\substack{\mu \in \Lambda(n, r) \\ \nu \in \Lambda(n, s) \\ \lambda=\mu-\nu}} M^{\mu} \otimes M^{\nu}
$$

A more explicit description of these weight spaces as $\mathcal{B}_{r, s}^{(n)}$-modules can be given in the case $r=s$. Indeed there is an isomorphism of $\mathfrak{g l}_{n}$-bimodules $V^{d, d} \rightarrow \bigotimes^{d} \mathfrak{g l}_{n}$ defined

$$
v_{i_{1}} \otimes \cdots \otimes v_{i_{d}} \otimes v_{-j_{1}} \otimes \cdots \otimes v_{-j_{d}} \mapsto e_{i_{1}, j_{1}} \otimes \cdots \otimes e_{i_{d}, j_{d}}
$$

A basis element $v=e_{i_{1}, j_{1}} \otimes \cdots \otimes e_{i_{d}, j_{d}}$ of $\otimes^{d} \mathfrak{g l}_{n}$ corresponds to a dissection

$$
T_{v}=\left\{T_{v}^{11}, \ldots, T_{v}^{1 n}, T_{v}^{21}, \ldots, T^{n n}\right\}, \quad \text { where } T_{v}^{i j}=\left\{k \mid e_{i_{k}, j_{k}}=e_{i j}\right\}
$$

Hence the $\lambda$-weight space, $N^{\lambda}$, of $\bigotimes^{d} \mathfrak{g l}_{n}$ has a basis of $A$-tabloids, where

$$
A \in \bigcup_{\substack{\mu, \nu \in \Lambda(n, d) \\ \lambda=\mu-\nu}} A_{\nu}^{\mu} .
$$

A cute exercise we leave with the reader is to describe the $\mathcal{B}_{d, d}^{(n)}$ action on these tabloids.

\section{REFERENCES}

[AR17] Cosima Aquilino, Rebecca Reischuk. The monoidal structure on strict polynomial functors. J. Algebra 485:213-229, 2017 arXiv:1503.05108v1

[BCHLLS94] Georgia Benkart, Manish Chakrabarti, Thomas Halverson, Robert Leduc, Chanyoung Lee, Jeffrey Stroomer. Tensor product representations of general linear groups and their connections with Brauer algebras. J. Algebra 166:529-567, 1994

[Bra37] Richard Brauer. On algebras which are connected with the semisimple continuous groups. Annals of Mathematics 38:857-872, 1937

[Bro56] William Brown. The semisimplicity of $\omega_{f}^{n}$. Annals of Mathematics 63:324-335, 1956 
[CKM14] Sabin Cautis, Joel Kamnitzer, Scott Morrison. Webs and quantum skew Howe duality. Mathematische Annalen 360:351-390, 2014 arXiv:1210.6437v4

[DGS06] Stephen Doty, Anthony Giaquinto, John Sullivan. Presenting Schur algebras in types B, C, D. Advances in Mathematics 206:434-454, 2006 arXiv:math/0504075v1

[DGS09] Stephen Doty, Anthony Giaquinto, John Sullivan. On the defining relations for generalized q-Schur algebras. Advances in Mathematics 221:955-982, 2009 arXiv:0801.0005v3

[Dot03] Stephen Doty. Presenting generalized q-Schur algebras. Representation Theory 7:196-213 (electronic), 2003. arXiv:math/0305208v1

[Dot12] Stephen Doty. A characteristic-free decomposition of tensor space as a Brauer algebra module. J. Lie Theory 22(3):869-882, 2012 arXiv:1104.4316v1

[Don86] Stephen Donkin. On Schur Algebras and Related Algebras, I. J. Algebra 104(2):310-328, 1986

[JK81] Gordon Douglas James, Adalbert Kerber. The Representation Theory of the Symmetric Group, volume 16 of Encyclopedia of Mathematics and its Applications. Addison-Wesley, Reading, MA, 1981

[ST17] Antonio Sartori, Daniel Tubbenhauer. Webs and $q$-Howe dualities in types BCD. 2017 arXiv:math/1701.02932

School of Mathematics and Statistics, University of Sydney, Australia

E-mail address: g.wiggins@maths.usyd.edu.au 